\title{
Review Article \\ Cognitive Radio Transceivers: RF, Spectrum Sensing, and Learning Algorithms Review
}

\author{
Lise Safatly, ${ }^{1}$ Mario Bkassiny, ${ }^{2}$ Mohammed Al-Husseini, ${ }^{3}$ and Ali El-Hajj ${ }^{1}$ \\ ${ }^{1}$ ECE Department, American University of Beirut, Beirut 1107 2020, Lebanon \\ ${ }^{2}$ ECE Department, State University of New York, Oswego, NY 13126, USA \\ ${ }^{3}$ Beirut Research \& Innovation Center, Lebanese Center for Studies \& Research, Beirut 2030 8303, Lebanon
}

Correspondence should be addressed to Lise Safatly; les12@aub.edu.lb

Received 13 November 2013; Revised 14 January 2014; Accepted 17 January 2014; Published 24 March 2014

Academic Editor: Said E. El-Khamy

Copyright (C) 2014 Lise Safatly et al. This is an open access article distributed under the Creative Commons Attribution License, which permits unrestricted use, distribution, and reproduction in any medium, provided the original work is properly cited.

\begin{abstract}
A cognitive transceiver is required to opportunistically use vacant spectrum resources licensed to primary users. Thus, it relies on a complete adaptive behavior composed of: reconfigurable radio frequency (RF) parts, enhanced spectrum sensing algorithms, and sophisticated machine learning techniques. In this paper, we present a review of the recent advances in CR transceivers hardware design and algorithms. For the RF part, three types of antennas are presented: UWB antennas, frequency-reconfigurable/tunable antennas, and UWB antennas with reconfigurable band notches. The main challenges faced by the design of the other RF blocks are also discussed. Sophisticated spectrum sensing algorithms that overcome main sensing challenges such as model uncertainty, hardware impairments, and wideband sensing are highlighted. The cognitive engine features are discussed. Moreover, we study unsupervised classification algorithms and a reinforcement learning (RL) algorithm that has been proposed to perform decisionmaking in CR networks.
\end{abstract}

\section{Introduction}

Cognitive radio (CR) provides a solution to the inefficient use of the frequency spectrum [1-3]. This inefficiency is due to the current radio spectrum regulations which assign specific bands to particular services and grant licensed bands access to only licensed users. CR implements dynamic spectrum allocation policies by allowing unlicensed users (secondary users) to access spectrum bands licensed to primary users while avoiding interference with them $[2,3]$. This necessitates at the RF front end more constraints on the antenna design, the development of algorithms for sensing the surrounding environment, and autonomously adapting to particular situations through a cognitive engine $[1,4-9]$. These three design elements are introduced in this section and presented in more detail in the rest of this paper.

Spectrum underlay and spectrum overlay represent the two main approaches of sharing spectrum between primary users (PUs) and secondary users (SUs). The underlay approach imposes constraints on the transmission power, which can be satisfied using ultrawideband antennas (UWB).
UWB antennas are also used for channel sensing in overlay CR but must be frequency reconfigurable or tunable. In this case, a single-port antenna can have UWB response for sensing and can be reconfigured for tunable narrowband operation when needed to communicate over a white space. It is also possible to use dual-port antennas for overlay CR, in which one port has UWB frequency response and is used for channel sensing, and the second port is frequencyreconfigurable/tunable and used for communicating. In a third possible spectrum sharing approach, the antennas could be UWB antennas but should have the ability to selectively induce frequency notches in the bands used by PUs, thus avoiding any interference to them and giving the UWB transmitters of the SUs the chance to achieve long-distance communication.

Aside from the antenna, the design of the other RF blocks faces main challenges related to the ADC/DAC, the dynamic range or range of signal strengths to deal with, to the linearity of low-noise amplifiers (LNAs), and to the frequency agility of the duplexer and filters. 
In CR, smart transceivers scan the spectrum in order to find white spaces and transmit adaptive signals. This functionality requires sophisticated algorithms to overcome practical imperfections such as model uncertainty and hardware nonideality. Some of the well-known spectrum sensing algorithms are energy detection [10-12], matched filterbased detection [11, 13], cyclostationarity-based detection [14-18], covariance-based sensing $[19,20]$, and eigenvaluebased sensing [21-23]. Blind detectors were also introduced to elude the model uncertainty problem. Spectrum sensing algorithms could be affected by RF impairments by inducing unwanted frequency components in the collected signal spectrum. The effects of such impairments are reduced through a postprocessing of the signal [24-26]. In addition, a robust detector based on smart digital signal processing lowers the effects of RF impairments and guarantees a high sensing accuracy.

The concept of cognitive radios (CRs) goes beyond dynamic spectrum access (DSA) applications and aims to improve the quality of information (QoI) of users [5]. This requires an intelligent radio that uses spectrum sensing techniques to observe the RF activities and is able to autonomously adapt to particular situations [9]. This is achieved through a reasoning engine which executes actions based on certain hard-coded rules and strategies [27]. Hardcoded policies are completely specified by the system designer and may result in the desired performance as long as the operating conditions do not deviate from the original assumed model. However, due to unexpected changes in the RF environment, the hard-coded rules may not lead to optimal performance, making them inefficient in this case. Cognitive radios, however, can overcome this problem by updating or augmenting their own sets of policies and rules based on past experience [27], which may lead to a more reliable communication performance $[9,27]$. This makes the learning ability a fundamental building block of any CR to achieve autonomous intelligent behavior [9, 27-29].

In this paper, Section 2 presents the antenna designs for CR: ultrawideband (UWB) antennas, antennas with reconfigurable band rejection, and frequency-reconfigurable or tunable antennas. It also briefly discusses the challenges faced by the design of RF blocks for CR devices. Section 3 discusses spectrum sensing by presenting challenges, novel solutions, and spectrum sensing algorithms. RF imperfections and wideband sensing are also studied and recent blind detectors, robust algorithms, and wideband techniques are presented. Section 4 presents a cognitive engine and several unsupervised classification algorithms for autonomous signal classification in CRs, and a reinforcement learning (RL) algorithm that performs decision-making in CR networks. Conclusive remarks are given in Section 6.

\section{RF Frontends for Cognitive Radio}

CR transceivers are required to look for and operate in white spaces, which could exist anywhere inside a wide frequency range. This comes different from conventional wireless transceivers which are bound to certain preallocated frequency bands. As a result, significant challenges have to be dealt with when designing the RF components of a CR transceiver, such as the antennas, the power amplifiers (PAs), the filters/duplexers, and the analog-to-digital and digital-toanalog converters (ADCs/DACs).

2.1. Antennas for Cognitive Radio. Two main approaches of sharing spectrum between primary users (PUs) and secondary users (SUs) exist: spectrum underlay and spectrum overlay. In the underlay approach, SUs should operate below the noise floor of PUs, and thus contingent constraints are imposed on their transmission power. Ultrawideband (UWB) technology is very suitable as the enabling technology for this approach. In spectrum overlay CR, SUs search for unused frequency bands, called white spaces, and use them for communication.

UWB antennas are used for underlay CR and also for channel sensing in overlay CR. For communication in overlay $\mathrm{CR}$, the antenna must be frequency reconfigurable or tunable. Single- and dual-port antennas for overlay CR can be designed. In the dual-port case, one port has UWB frequency response and is used for channel sensing, and the second port, which is frequency reconfigurable/tunable, is used for communicating. In the more challenging single-port design, the same port can have UWB response for sensing and can be reconfigured for tunable narrowband operation when required to communicate over a white space.

A third possible spectrum sharing approach results from the use of the UWB technology in an overlay scheme. Here, the antennas could basically be UWB antennas but should have the ability to selectively induce frequency notches in the bands used by PUs, thus avoiding any interference to them and giving the UWB transmitters of the SUs the chance to increase their output power and hence to achieve longdistance communication.

A concise review of antenna designs for cognitive radio, covering the above three antenna types, is given in [30].

2.1.1. UWB Antennas. UWB antennas were originally meant to radiate very short pulses over short distances. They have been used in medical applications, GPRs, and other shortrange communications requiring high throughputs. The literature is rich with articles pertaining to the design of UWB antennas [31-41]. For example, the authors in [31] present a unidirectional UWB antenna based on a full-ground plane. To keep this full-ground plane, they sequentially employ a list of broadbanding techniques: (1) resonance overlapping, (2) slot, (3) parasitic patch, (4) Vivaldi blending, (5) stepped notch, and (6) rectangular and T-shape slits. The resulting antenna has an impedance bandwidth from $3.6 \mathrm{GHz}$ to $10.3 \mathrm{GHz}$ while keeping the unidirectionality of the radiation pattern.

In general, the guidelines to design UWB antennas include the following.

(i) The proper selection of the patch shape. Round shapes and round edges lead to smoother current flow and,as a result, to better wideband characteristics. 


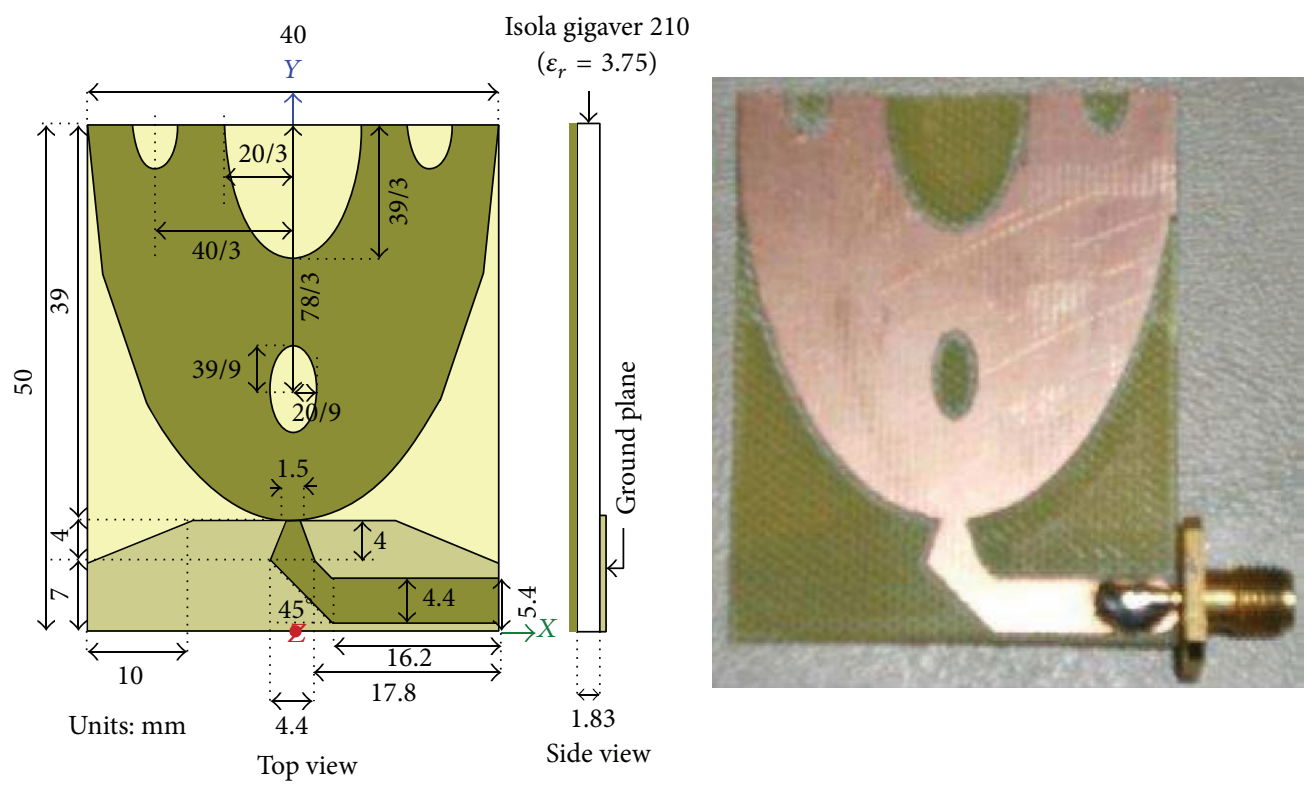

FIGURE 1: Configuration and photo of the UWB antenna in [42]. The antenna combines several bandwidth enhancement techniques.

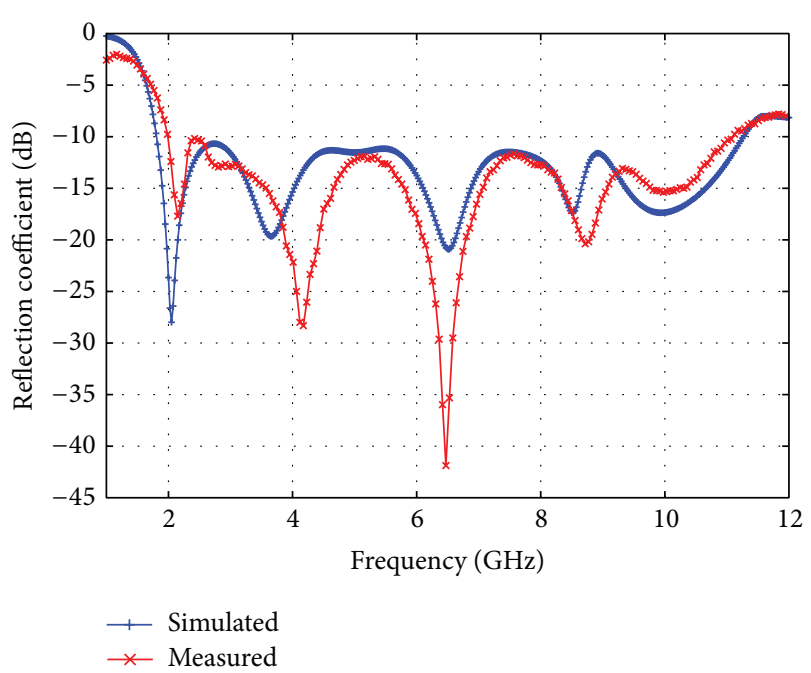

Figure 2: Reflection coefficient of the UWB antenna in Figure 1.

(ii) The good design of the ground plane. Partial ground planes, and ground planes with specially designed slots, play a major role in obtaining UWB response. Keeping a full-ground plane is possible, but in that case an elaborate work has to be done on the patch design.

(iii) The matching between the feed line and the patch. This is achieved using either tapered connections, inset feed, or slits under the feed in the ground plane.

(iv) The use of fractal shapes, which are known for their self-repetitive characteristic, used to obtain multiand wideband operation, and their space-filling property, which leads to increasing the electrical length of the antenna without tampering with its overall physical size.
Combinations of these guidelines were used in the following two examples. The UWB design presented in [42] features a microstrip feed line with two $45^{\circ}$ bends and a tapered section for size reduction and matching, respectively. The ground plane is partial and comprises a rectangular part and a trapezoidal part. The patch is a half ellipse with the cut made along the minor axis. Four slots whose location and size relate to a modified Sierpinski carpet, with the ellipse as the basic shape, are incorporated into the patch. The geometry of this antenna is shown in Figure 1. Four techniques are applied for good impedance matching over the UWB range: (1) the specially selected patch shape, (2) the tapered connection between the patch and the feed line, (3) the optimized partial ground plane, and (4) the slots whose design is based on the knowledge of fractal shapes. As a result, this antenna has an impedance bandwidth over the $2-11 \mathrm{GHz}$ range, as shown in Figure 2, and thus can operate in the bands used for UMTS, WLAN, WiMAX, and UWB applications. It has omnidirectional radiation patterns due to the partial ground plane.

The effect of the ground plane on the performance of UWB antennas is studied in [43]. Herein, it is proven that it is possible to obtain an ultrawide impedance bandwidth using either a partial ground plane or a ground plane with an optimized large slot, where in both cases the same exact patch is used. This design has a coplanar-waveguide feed that connects to an egg-shaped radiator. A photo of both versions is given in Figure 3.

2.1.2. Antennas with Reconfigurable Band Rejection. As previously stated, UWB technology is usually associated with the CR underlay mode. It can, however, be implemented in the overlay mode. The difference between the two modes is the amount of transmitted power. In the underlay mode, UWB has a considerably restricted power, which is spread over a wide frequency band. In the overlay mode, however, 


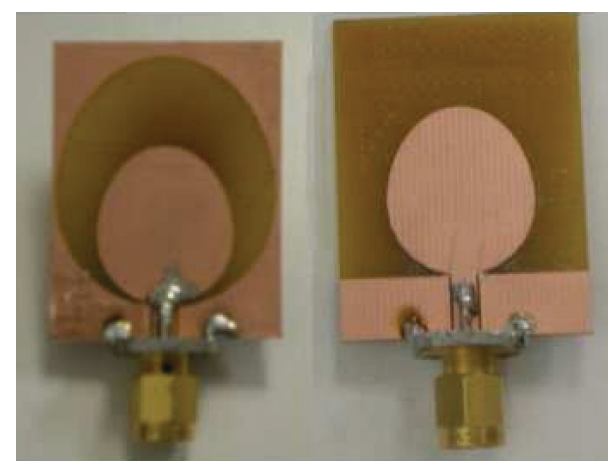

Figure 3: Two UWB antennas with optimized ground planes [43]. One has a partial ground plane and the other has a ground plane with a large slot.

the transmitted power can be much higher. It actually can be increased to a level that is comparable to the power of licensed systems, which allows for communication over medium to long distances. But this mode is only applicable if two conditions are met: (1) if the UWB transmitter ensures that the targeted spectrum is completely free of signals of other systems, or if it shapes its pulse to have nulls in the bands used by these systems, and (2) if the regulations are revised to allow for this mode of operation [44]. Pulse adaptation for overlay UWB CR has been discussed in [45]. UWB can also operate in both underlay and overlay modes simultaneously. This can happen by shaping the transmitted signal so as to make part of the spectrum occupied in an underlay mode and some other parts occupied in an overlay mode. In the overlay UWB scenario, the antenna at the front end of the CR device should be capable of operating over the whole UWB range, for sensing and determining the bands that are being used by primary users, and should also be able to induce band notches in its frequency response to prevent interference to these users. Even if the UWB power is not increased having these band notches prevent raising the noise floor of primary users.

Antennas that allow the use of UWB in overlay CR should have reconfigurable band notches. Several band-notching techniques are used in such antennas, the most famous of which is the use of split-ring resonators (SRRs) [46] and the complementary split-ring resnators (CSRRs) [47]. SRRs have attracted great interest among electromagneticians and microwave engineers due to their applications to the synthesis of artificial materials (metamaterials) with negative effective permeability. From duality arguments, CSRRs, which are the negative image of SRRs and roughly behave as their dual counterparts, can generate a negative permittivity media.

Some recent UWB antenna designs with fixed band notches are reported in [48-55]. The works in [56-59] are for UWB antennas with reconfigurable band notches.

A UWB design with a single reconfigurable band notch is proposed in [60]. The configuration of this design and a photo of its fabricated prototype are shown in Figure 4. Originally, the antenna is a UWB monopole based on a microstrip line feed and a partial ground plane. The patch is rectangular with rounded corners. A slit is etched in the ground below the feed, for better matching. As a result, this antenna has an impedance bandwidth that covers the whole UWB frequency range. Four nested CSRRs are incorporated in the patch. Three electronic switches are mounted across the slots. The sequential activation (deactivation) of the switches leads to the functioning of a larger (smaller) CSRR and thus results in a notch at a lower (higher) frequency. The following switching cases are considered: Case 1 when all three switches are $\mathrm{ON}$, Case 2 when only S3 is deactivated, Case 3 when only S1 is ON, and finally Case 4 when all switches are OFF. The resulting reflection coefficient plots, corresponding to the different switching states, are shown in Figure 5. The plots show one notch, which can occur in one of 3 bands or can completely disappear. In the latter case, the antenna retrieves its UWB response, which enables it to sense the whole UWB range.

The antenna reported in [61] is capable of concurrently inducing three band notches, which are independently controllable, using only three RF switches. This is done using three CSRRs etched on the patch. There are eight switching cases for this design, with one of them being the original UWB no-notch case. A UWB antenna with reconfigurable band notches can also be designed by incorporating a bandstop filter in the feed line of a UWB antenna. With this structure, the switching elements will be mounted on the feed line, away from the radiating patch, which makes the bias circuit of the switches simpler to design. Such a filter antenna (filtenna) with two reconfigurable rejection bands is presented in [62]. Its structure is shown in Figure 6. The UWB antenna is based on a rounded patch and a partial rectangular ground plane. A reconfigurable filter with two stop bands is incorporated along its microstrip feed line. The filter is based on one rectangular single-ring CSRR etched on the line and two identical rectangular single-ring SRRs placed in close proximity to it. The resonance of the CSRR is controlled via a switch and that of the two SRRs via two switches that are operated in parallel. As a result, there are four switching scenarios. The resulting reflection coefficient plots are shown in Figure 7. Case 1, where no band notches exist, allows the antenna to sense the UWB range to determine the narrowband primary services that are transmitting inside the range. In the other three cases, the notches block the UWB pulse components in the $3.5 \mathrm{GHz}$ band, the $5.5 \mathrm{GHz}$ band, or both. It should be noted that notches due to the SRRs and the CSRR around the feed are stronger than those due to CSRRs or any notching structures implemented in the patch. This is because energy is concentrated in a smaller area in the feed and coupling with the SRRs/CSRR is higher. Due to the location of the switches, connecting the DC bias lines, especially to the SRRs, which are DC-separated from anything else, is an easy task. A wire can be used to drive the switch on the CSRR. A note is that extra band notches can be obtained by placing more SRRs around the feed line. Tunable versions of these notches can be obtained by replacing the RF switches with varactors.

2.1.3. Frequency-Reconfigurable/Tunable Antennas. Antennas designed for overlay CR should have the capability to sense the channel and communicate over a small portion 

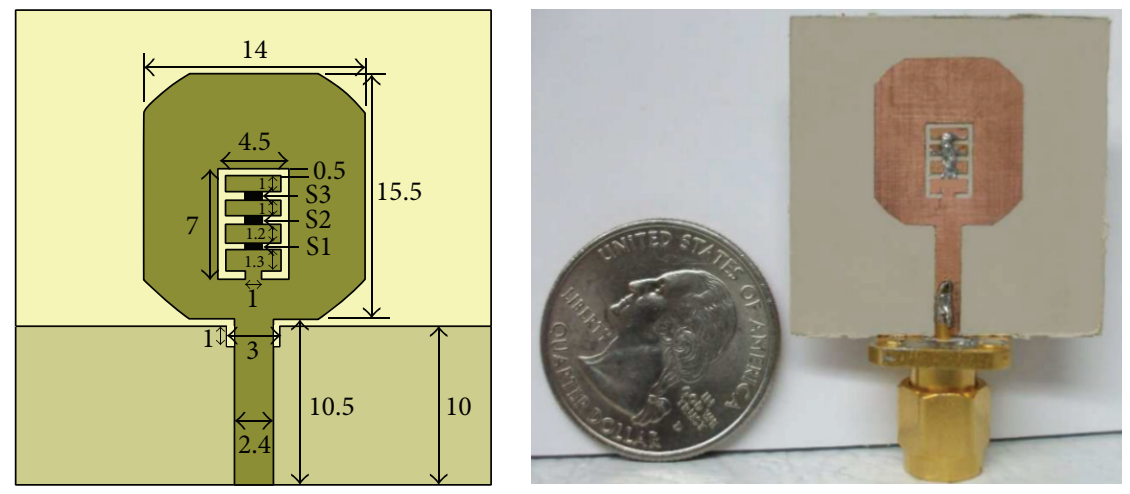

FIGURE 4: Geometry and photo of an antenna with one reconfigurable rejection band [60].

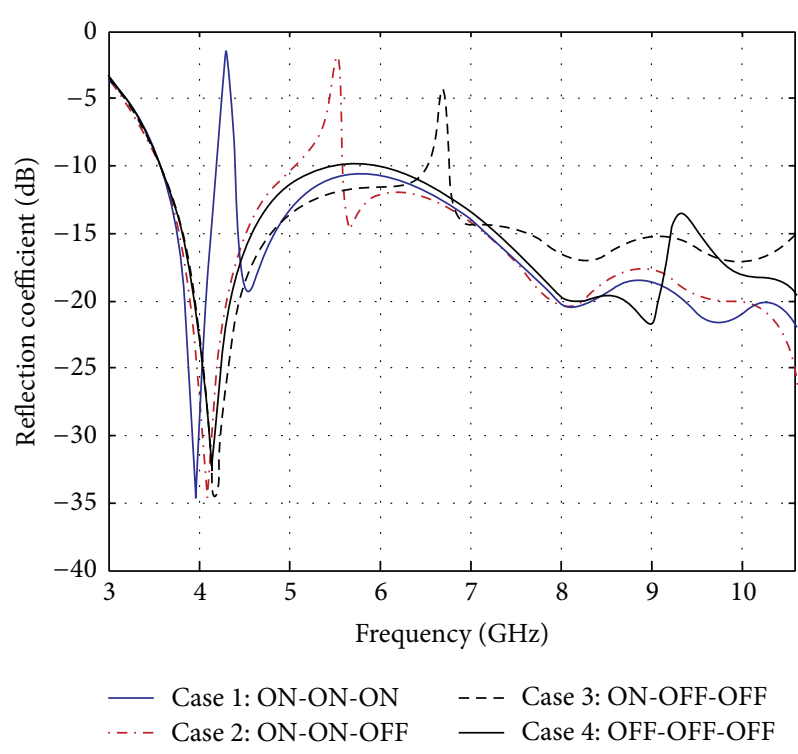

FIGURE 5: Reflection coefficient for the different switching cases of the antenna in Figure 4.

of it. These antennas can be implemented as dual port, where one port is UWB and the other is narrowband and frequency reconfigurable. They can also be designed as single port, where the same port is used for both sensing and communicating, and thus should switch between wideband and narrowband operations. For the dual-port designs, the isolation between the two ports is an issue. More elaborate work is required for single-port designs.

A dual-port antenna for overlay was proposed in [63]. Its structure consists of two printed antennas, namely, wide- and narrowband antennas. The design in [64] also combines wide and narrow band antennas, where the wideband one covers the $2.6-11 \mathrm{GHz}$ range and the narrowband one is tunable, using a varactor, between $6.85 \mathrm{GHz}$ and $7.20 \mathrm{GHz}$. A simple dual-port design is presented in [65]. The configuration of this design, which comprises two microstrip-line-fed monopoles sharing a common partial ground, is shown in Figure 8. The sensing UWB antenna is based on an eggshaped patch. The UWB response of the sensing antenna is guaranteed by the design of the patch, the partial ground plane, and a feed matching section. The communicating antenna is a simple microstrip line matched to the $50-\Omega$ feed line. Two electronic switches are incorporated along this line. Controlling these switches leads to various resonance frequencies within the UWB range, as shown in Figure 9 for three considered switching cases.

Dual-port antennas enable simultaneous sensing and communicating over the channel, but have limitations in terms of their relatively large size, the coupling between the two ports, and the degraded radiation patterns. These limitations are solved by the use of single-port antennas, but these are only suitable when the channel does not change very fast, and thus sensing and communication are possible, sequentially.

Single-port reconfigurable wideband/narrowband antennas are reported in [66-68]. The design in [66] has a wide bandwidth mode covering the $1.0-3.2 \mathrm{GHz}$ range and three narrowband modes within this range. In [67], fifteen PIN diode switches are used on a single-port Vivaldi antenna, leading to a wideband operation over the $1-3 \mathrm{GHz}$ band and six narrowband states inside this range. GaAs field-effect transistor (FET) switches are used in [68] to connect multiple stubs of different lengths to the main feed line of a UWB circular-disc monopole. The result is an antenna that can be operated in a UWB mode or in a reconfigurable narrowband mode over one of three frequency subbands: the first covers $2.1-2.6 \mathrm{GHz}$, the second covers $3.6-4.6 \mathrm{GHz}$, and finally the third covers a dual band of $2.8-3.4 \mathrm{GHz}$ and $4.9-5.8 \mathrm{GHz}$. The design in [69] is a filtenna based on a reconfigurable bandpass filter embedded in the feed line of a UWB monopole. A UWB and five narrowband operation modes characterize this design.

A printed Yagi-Uda antenna tunable over the 478$741 \mathrm{MHz}$ UHF TV band is presented in [70], where the narrowband frequency tunability is obtained by loading the driver dipole arms and four directors with varactor diodes. A miniaturized tunable antenna for TV white spaces is reported in [71]. In [72], two PIN diodes and two varactors are employed for narrow band tuning between 1.39 and $2.36 \mathrm{GHz}$. A Vivaldi-based filtenna with frequency tunability over the $6.1-6.5 \mathrm{GHz}$ range is presented in [73]. The singleport designs in $[70,72,73]$ are only capable of narrowband operation, which means that wideband spectrum sensing has 

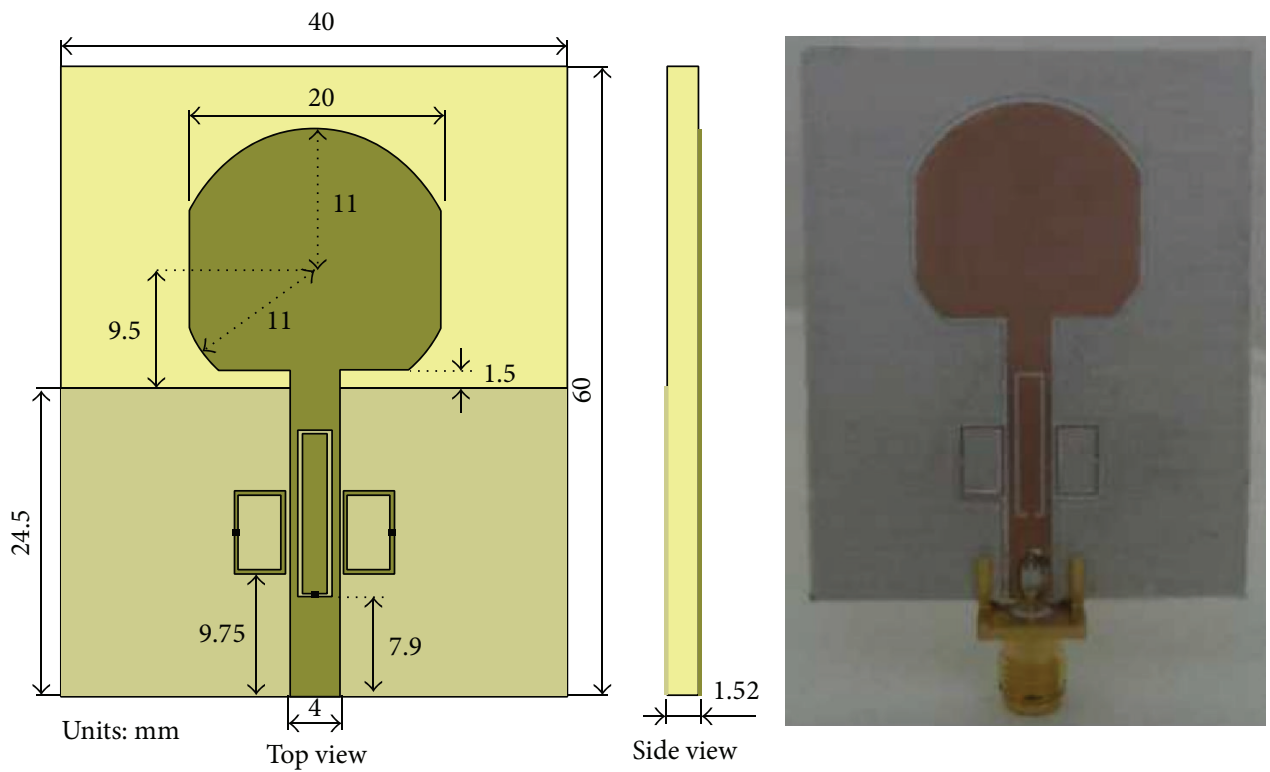

FIGURE 6: Geometry and photo of a filter antenna with two reconfigurable band notches [62].

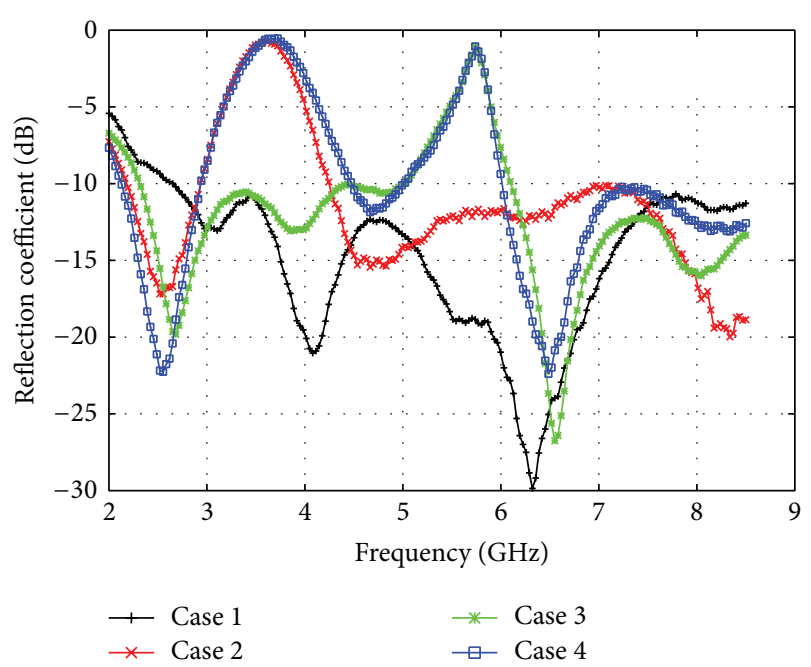

Figure 7: Reflection coefficient for the different switching cases of the antenna in Figure 6.

to be done progressively, a narrow chunk of frequencies at a time. This is also the case with the filtenna design shown in Figure 10. Here, a tunable bandpass filter is embedded along the microstrip feed line of a UWB monopole antenna, where the filter is based on a T-shaped slot incorporated in the microstrip line between a pair of gaps. For the purpose of achieving frequency tunability, a varactor is included in the design, as indicated. Changing the capacitance of the varactor changes the notch band, caused by the T-shaped slot, and as a result the narrow passband of the overall filter. The DC lines of the varactors are connected with ease. Due to the presence of the two gaps, DC is separated from both the antenna port and the patch. Two surface-mount inductors are used over the DC lines as RF chokes. The computed reflection coefficient plots are given in Figure 11. They show narrowband tunability over the 4.5-7 GHz frequency range, for capacitance values between 0.3 and $7 \mathrm{pF}$.

2.2. RF Design Challenges. The design challenges for the RF section of a CR transceiver are well reviewed in [74-76]. Diagrams of a CR transceiver architecture appear in [74]. For wideband operation, such as that required for sensing, the main challenges relate to the ADC/DAC, the dynamic range, or range of signal strengths to deal with, which could be as wide as $100 \mathrm{~dB}$, to the linearity of low-noise amplifiers (LNAs), which has to be high, and to achieve impedance matching over a wide frequency range. For tunable narrowband operation, the key issues are the frequency agility of the duplexer and filters.

2.2.1. ADCs and DACs. In many situations, the desired signal received by wireless device could sometimes be $100 \mathrm{~dB}$ weaker than other in-band signals generated by nearby transmitters of the same communication standard or some out-of-band blockers caused by any transmitter. This would demand a dynamic range of about $100 \mathrm{~dB}$ on the ADC. Ideally, the RF signals received by a CR system should be digitized as close to the antenna as possible, so that all the processing is done at the level of the digital signal processor (DSP). In this case, the $\mathrm{ADC}$ and $\mathrm{DAC}$ should have the $100 \mathrm{~dB}$ dynamic range, explained earlier, be operable over a UWB frequency range and handle significant levels of power. These requirements are still far beyond the limits of available technology, that is why the more realistic $\mathrm{CR}$ receivers reduce both the required dynamic range and the conversion bandwidth by having downconversion and filtering functions implemented before ADC.

On the path towards ideal CR receivers, several ADCs can be used in parallel to widen the conversion bandwidth. In [77], a parallel continuous time $\Delta \Sigma \mathrm{ADC}$ is presented, which 

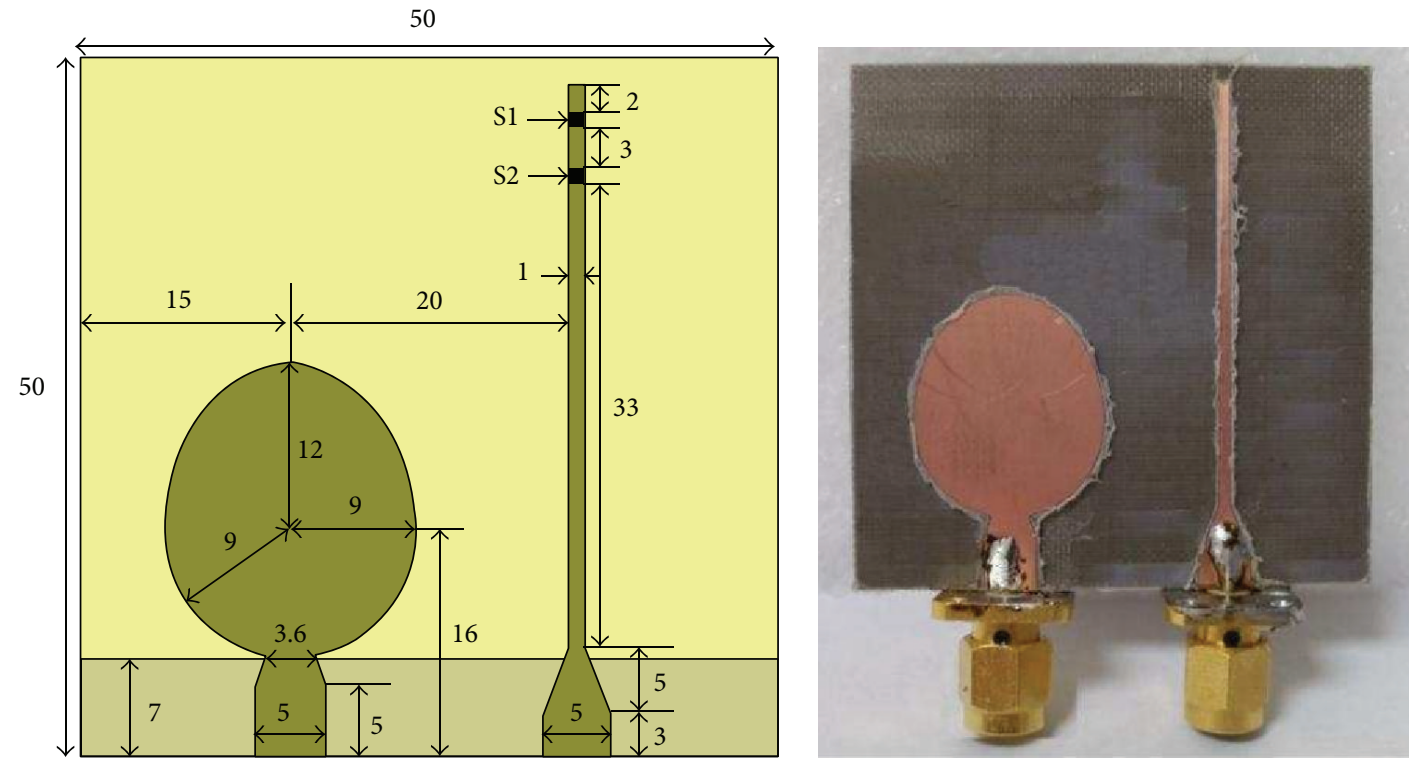

Units: $\mathrm{mm}$

FIGURE 8: Geometry and photo of the dual-port ultrawideband-narrowband antennas in [65].
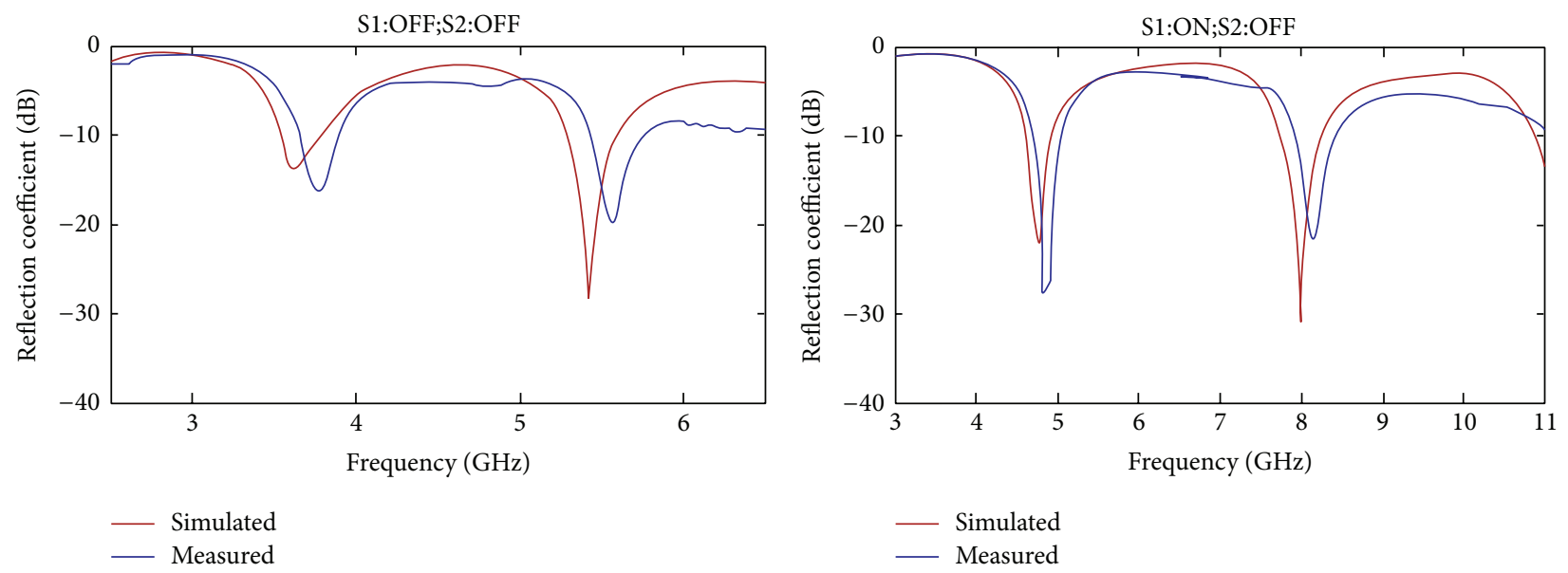

(a)

(b)

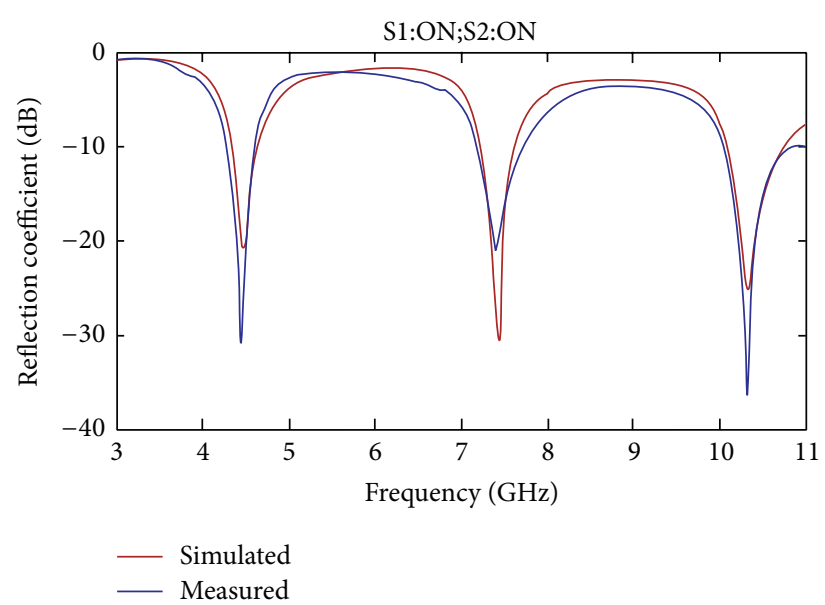

(c)

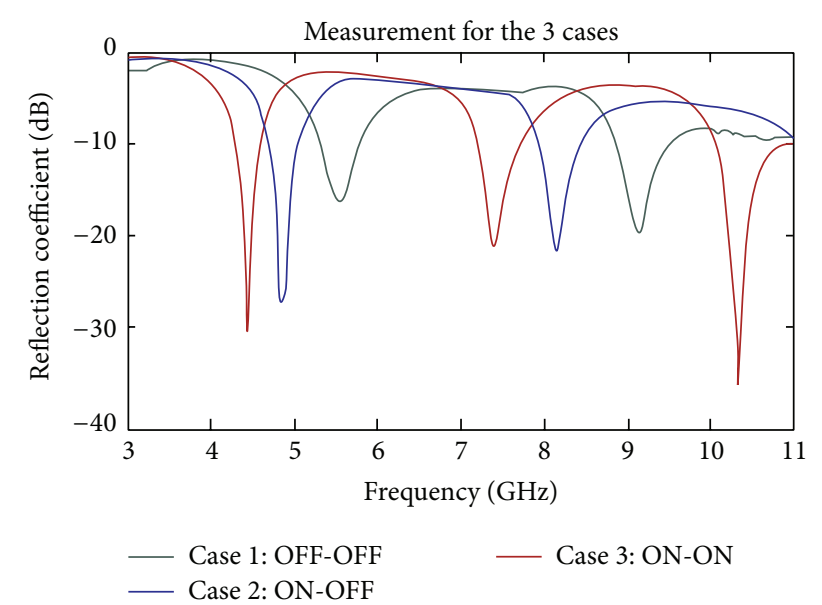

(d)

FIGURE 9: Reflection coefficient for 3 selected switching cases of the antenna in Figure 8. 

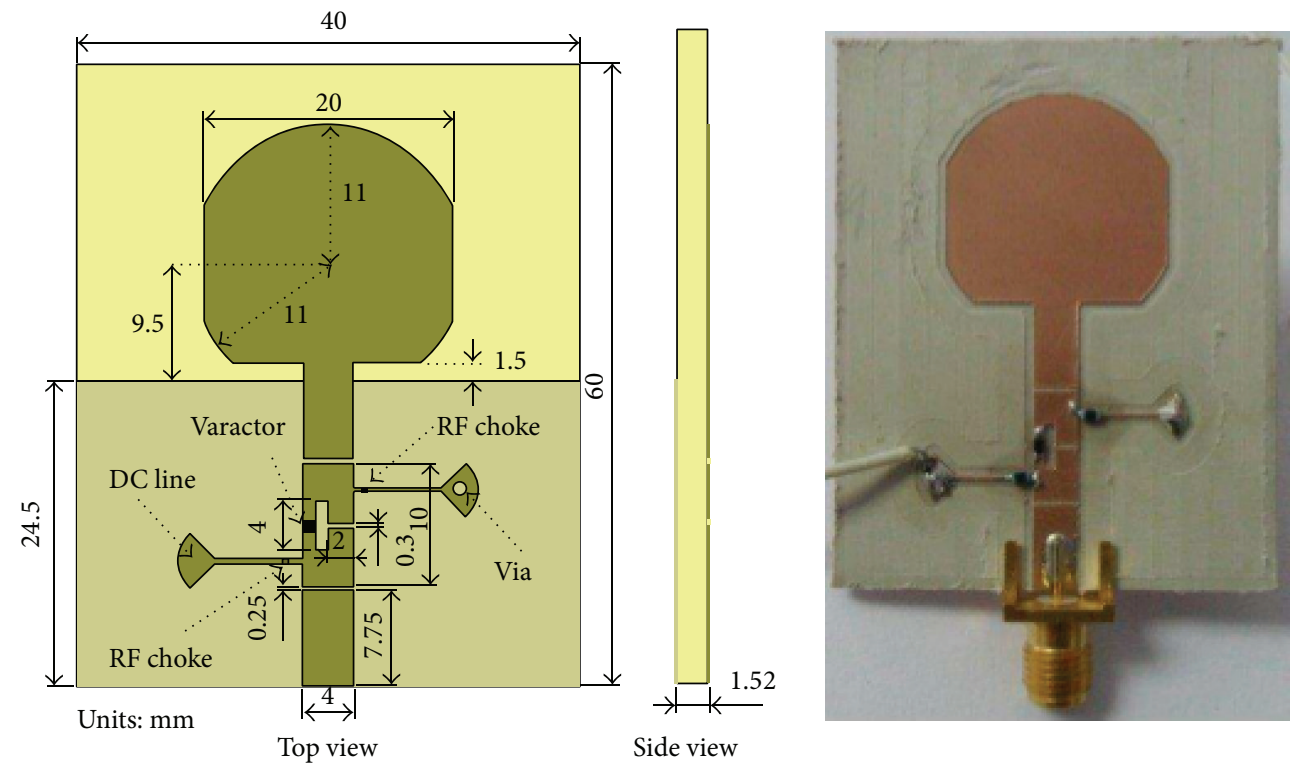

FIGURE 10: Geometry and photo of a tunable narrowband filtenna.

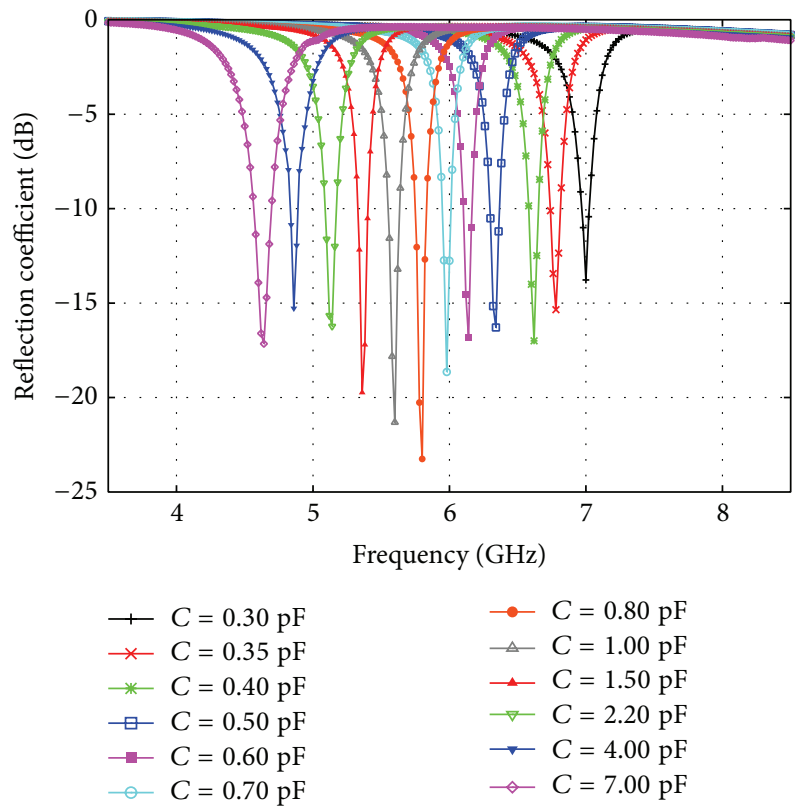

FIGURE 11: Reflection coefficient of the filtenna in Figure 10 for the indicated varactor capacitance values.

requires very complex digital synthesis filters. The hybrid filter bank-based ADC presented in [78] could be a promising solution for CR applications, but its use necessitates high resource calibration since it is sensitive to analog filter errors and imperfections. A solution that provides good performance in terms of speed employs time interleaving [79], but it lacks the resolution and dynamic range. To suppress low order nonlinearities of parallel ADCs and nonlinearities caused by pre-ADC analog components, gigital postlinearization is desired. The technique in $[80,81]$ combines time multiplexing and frequency multiplexing by using bandpass charge sampling filters as analysis filters in hybrid filter banks architecture. This leads to reduced complexity of analog analysis filters and simultaneously of the sensitivity to analog errors and imperfections. Yet, the practical implementation with the aim of widening the bandwidth and sensitivity still requires a deeper investigation.

RF DACs are utilized in the fully digital RF transmitters presented in $[82,83]$. The design of these DACs is less challenging than that of ADCs, although more improvements are still necessary.

2.2.2. Low-Noise Amplifier. A receiver's performance is primarily determined by the linearity and adequate matching over a wide frequency range of the broadband or tunable LNA. A broadband LNA topology achieving a frequency range of $50 \mathrm{MHz}$ to $10 \mathrm{GHz}$ was proposed in [76]. The input capacitance is canceled by the LNA using inductive behavior provided by negative feedback. Advanced CMOS technologies make it easier to design such high-frequency LNAs without any large inductors. On the other hand, the linearity issue becomes crucial because the supply voltage for core transistors decreases to around $1 \mathrm{~V}$ in $90 \mathrm{~nm}$ or more advanced processes, which results in a limited voltage swing. LNAs using thick-oxide transistors may prevent this problem [84]. The mixer-first RF front ends $[85,86]$ are another approach for improving linearity performance. An impedance translation technique, which uses passive mixers followed by capacitive loads, can provide low impedance for out-of-band blockers $[87,88]$, which makes it attractive.

The even-order nonlinearity of the LNA also should be considered in wideband CR systems. This is because secondorder intermodulation (IM2) products generated by an LNA can fall within the CR band, thus corrupting the desired signal even before downconversion. A differential LNA topology seems attractive here, but a balun is necessary if the antenna is single ended. Design of low-loss baluns operating across two or three decades of bandwidth is challenging. A useful balun-LNA topology is proposed in [89]. 
2.2.3. Downconversion Mixer. Another issue in the wideband operation of a CR device is harmonic mixing. The RF signal is often downconverted using square-wave local-oscillator (LO) signals that contain large odd-order harmonic components. This is done to obtain flat performances of noise and gain across a wide frequency range. The use of a harmonicrejection mixer (HRM) is one way to remove odd-order LO harmonics. The authors in $[90,91]$ propose techniques to improve harmonic rejection ratios (HRRs). However, if seventh- and higher-order LO harmonics are to be rejected, the HRMs become much more complex.

2.2.4. Wideband Frequency Synthesizer. Multiple oscillators could be used to cover a wide frequency range. This solves the issue of the trade-off between tuning range and phase noises. For acceptable phase noise figures, the tuning range of inductor-capacitor (LC-) type oscillators is typically limited to about $\pm 15 \%$ at frequencies of tens of gigahertz. Frequency synthesizers for CR having two oscillators to support a wider frequency range are reported in $[84,92]$.

\section{Spectrum Sensing in Cognitive Radios}

In a CR scenario, secondary users are allowed to detect, exploit, and use underutilized spectral resources licensed to primary users. Such opportunistic behavior urges CR transceivers to scan the spectrum in order to find white spaces and transmit adaptive signals. Thus, spectrum sensing is the key function of smart receivers since it enables the cognitive cycle proposed by Mitola in [4, 93]. This functionality creates unique signal processing challenges and requires sophisticated algorithms to overcome practical imperfections such as model uncertainty and hardware imperfections.

To address these challenges, CR researchers proposed in the last decade various detectors that have different complexity levels, performance results, and requirements for implementation. Well-known spectrum sensing algorithms, sorted in an ascending order of complexity are energy detection (ED) [10-12], matched filter (MF-) based detection [11, 13], cyclostationarity-based detection (CSD) [14-18], covariancebased sensing $[19,20]$, and eigenvalue-based sensing [2123]. Some of these methods require a priori knowledge of noise and/or signal power information; these include $\mathrm{MF}$, CSD (relying on the full or partial knowledge of signal and noise levels), and ED (having a threshold dependent on the estimated noise power level). Blind detectors were recently proposed to elude the model uncertainty problem relying on advanced digital signal processing techniques.

In a cognitive receiver, RF impairments could harm the performance of the spectrum sensing algorithm by inducing unwanted frequency components in the collected signal spectrum. To mitigate the effects of such impairments, "Dirty $R F$ " is applied on the SU receiver inducing a postprocessing of the signal, thus compensating analog imperfections [24-26]. A robust detector, based on smart digital signal processing, should be able to digitally lower the effects of RF impairments and guarantee a high sensing accuracy.
The early-designed spectrum sensing algorithms aimed to detect a white space from narrow frequency bands where many emerging wireless applications require an opportunistic usage of a wideband spectrum [94-97]. Consequently, SUs are forced to scan a wide range of potential spectra and detect available holes to be able to transmit. One of the main concerns of the CR community is to conceive wideband spectrum sensing methods to replace the complicated implementation of high sampling rate ADCs, capable of downconverting wideband signals.

The selection of signal processing algorithms and their parameters reflects the speed and sensing time of the cognitive receiver. A complex signal processing algorithm should respect an optimum sensing value depending on the capabilities of the radio and its temporal characteristics in the environment. On the other hand, the ADC is considered as the primary bottleneck of the DSP architecture since it forces the clock speed of the system. Moreover, the selection of the digital signal processing platform affects the speed of the front end. All these parameters influence the sensing frequency and speed of cognitive radio receivers. For that, researchers focus on implementing sensing algorithms with low complexity, high speed, and flexibility in order to conceive an adaptive CR terminal.

In the following sections, we will describe three main practical spectrum sensing challenges and novel solutions. Model uncertainty, RF imperfections, and wideband sensing are studied and recent blind detectors, robust algorithms, and wideband techniques are presented. Accordingly, in the rest of this paper, we provide an overview of the state of the art of spectrum sensing algorithms that were proposed to answer these three major and hot research challenges.

3.1. Blind Detectors. As per regulation specifications, secondary users are required to detect very weak licensed users in order to protect primary transmissions [98, 99]. Any missed detection will enable an unlicensed transmission on a busy channel harming the incumbent primary signal. Unfortunately, many detectors reveal performance degradation at low SNR due to inappropriate estimation of the signal or noise models. This phenomenon is known as $\operatorname{SNR}$ wall $[10,100]$. For the ED, an estimation of the noise variance is required to select a suitable threshold. Imperfect knowledge of the noise model, especially in low SNR scenarios, will consequently deteriorate the efficiency of this algorithm. The SNR wall phenomenon also harms any detector based on the received signal's moments. Using cooperative spectrum sensing techniques or relying on calibration and compensation algorithms are possible solutions to the model uncertainty problem $[100,101]$. However, using totally blind detectors, which detect the presence of a signal without any knowledge of signal or noise parameters, is considered the ideal alternative. Two recently proposed blind detectors are described below.

3.1.1. Blind Eigenvalue-Based Detector. Zeng et al. devised a blind detector based on the computation of the minimum and maximum eigenvalues $\lambda_{\min }$ and $\lambda_{\max }$ of the sample covariance matrix $\mathbf{R}\left(N_{S}\right)$ defined in [22]. The test statistics 
Initialize: Acquire $L$ consecutive data samples and assume that there are $(M \geq 1)$ receivers (antennas), and $\left(N_{S}\right)$ is the total number of collected samples.

(a) Define the received vector $x_{n}$ given by: $\mathbf{x}(n)=\left[x_{1}(n), x_{2}(n), \ldots, x_{M}(n)\right]^{T}$

(b) The collection of $L$ consecutive outputs $\widehat{\mathbf{x}}_{n}$ is defined as: $\widehat{\mathbf{x}}_{n}=\left[\mathbf{x}^{T}(n), \mathbf{x}^{T}(n-1), \ldots, \mathbf{x}^{T}(n-L+1)\right]^{T}$

(c) Compute the sample covariance matrix $\mathbf{R}\left(N_{S}\right)$ : $\mathbf{R}\left(N_{S}\right)=\frac{1}{N_{S}} \sum_{n=L}^{L-1+N_{S}} \widehat{\mathbf{x}}_{n} \widehat{\mathbf{x}}_{n}^{H}$

(d) Compute $\lambda_{\max }$ and $\lambda_{\min }$ the maximum and minimum eigenvalues of the matrix $\mathbf{R}\left(N_{S}\right)$.

(e) Compute the threshold $v$ for the test statistics:

$v=\frac{\left(\sqrt{N_{S}}+\sqrt{M L}\right)^{2}}{\left(\sqrt{N_{S}}-\sqrt{M L}\right)^{2}}\left(1+\frac{\left(\sqrt{N_{S}}+\sqrt{M L}\right)^{-2 / 3}}{\left(N_{S} M L\right)^{1 / 6}} F_{1}^{-1}\left(1-P_{F A}\right)\right)$,

where $F_{1}$ is the Tracy-Widom distribution of order 1 [102].

The decision test:

(f) Decide on $H_{0}$ or $H_{1}$ by computing the ratio between $\lambda_{\max }$ and $\lambda_{\min }$ :

$\frac{\lambda_{\max }}{\lambda_{\min }} \gtrless_{H_{0}}^{H_{1}} v$

Algorithm 1: Steps of the MME detector.

of this maximum-minimum eigenvalue (MME) detection is simply given by

$$
\frac{\lambda_{\max }}{\lambda_{\min }} \gtrless_{H_{0}}^{H_{1}} v,
$$

where $v$ is the threshold calculated by using the number of acquired samples, the smoothing factor used for the calculation of $\mathbf{R}\left(N_{S}\right)$, and a selected probability of false alarm. It is expected that noise produces small eigenvalues, whereas the correlation inherited in modulated signals increases the eigenvalues. The proposed test statistic does not depend on any knowledge of noise, signal, or channel models; thus it is not sensitive to the model uncertainty problem. The detailed computational steps of this scheme are described in Algorithm 1.

3.1.2. The CAF Symmetry-Based Detector. This blind spectrum sensing detector is based on the symmetry property of the cyclic autocorrelation function (CAF). Benefiting from the sparsity property of CAF, the compressed sensing tool is adopted in this algorithm. A test statistic is defined, without the computation of any threshold, by checking if the estimated CAF exhibits symmetry or not. As demonstrated in [103], a positive symmetry check affirms the presence of a primary signal. The estimation of the cyclic autocorrelation vector is computed using an iterative optimization technique, called the orthogonal matching pursuit (OMP) [104]. The computational complexity of this algorithm is reduced by limiting the number of acquired samples and the number of needed iterations to ensure its practical feasibility. Algorithm 2 summarizes the main steps of this detector.

3.2. Robust Sensing Algorithms. In practice, CR receivers are composed of several sources of hardware imperfections such as low noise amplifiers (LNA), mixers, local oscillators (LO), and analog-to-digital converters (ADC). The most critical result of such impairments is the appearance of new frequency components in the received signal, classified in intermodulation distortions (IM), cross modulation (XM), and phase distortion (AM/PM). Consequently, strong primary users could harm the performance of traditional spectrum sensing techniques by adding unwanted spectrum components via front-end's nonlinearity. When these components overlap with weak secondary users, a degradation in the reliability of SU transmissions occurs. They can also virtually occupy the whole spectrum, thus decreasing the opportunity to find a vacant transmission band. In both scenarios, the accuracy of any proposed algorithm will be deteriorated. To mitigate these effects, a robust detector algorithm should be equipped with a compensation functionality to digitally reduce the effects of nonlinearities. Possible compensation algorithms could be based on feed-forward techniques with reference nonlinearity, feed-back equalization, and training symbol-based equalization. A well-known feed-forward technique to alleviate phase noise, carrier frequency offset, nonlinearities, I/Q imbalance, or ADC impairments is described below.

3.2.1. The AIC Algorithm. In [25], Valkama et al. devised the adaptive interference cancellation (AIC) algorithm, which is a feed-forward algorithm for the mitigation of second, third, and fifth order intermodulation distortion. The idea is to model the distortion caused by the interferer and then subtract them from the received signal. A mathematical formulation of the distortion model and order is studied before implementing the algorithm. Then, an imitation of the distortion products and an adaptive adjustment of their levels are performed to compensate the distorted signal. 
Initialize: Acquire $n$ data samples from the spectrum sensing interval formed by $N$ samples and set $(l+1)$ the number of OMP iterations, and $(M)$ the number of delays $\tau$ s

For $M$ different values of $\tau$,

(a) Calculate the autocorrelation vector $\mathbf{f}_{\tau_{0}}$ given by:

$\mathbf{f}_{\tau}=\left[f_{\tau}(0), f_{\tau}(1), \ldots, f_{\tau}(N-1)\right]^{T}$,

where $f_{\tau}(t)=y(t) y(t+\tau)$.

(b) Calculate the elements of the matrix $A$ performing the IDFT transform: $a_{(p, q)} e^{2 i \pi(p-1)(q-1) / N}$

The OMP algorithm

(c) Estimate the cyclic autocorrelation vector by solving the following system of equations: $A \mathbf{r}_{\tau}=\mathbf{f}_{\tau}$ by using an iterative optimization technique called

Orthogonal Matching Pursuit (OMP) that delivers an approximated solution $\widehat{\mathbf{r}}_{\tau}$. Symmetry check

(d) Calculate the symmetry index for this value of $\tau$, by ignoring the first amplitude that corresponds to the first iteration of OMP, and measuring the mean value of the abscissa of the remaining $(l-1)$ non zero elements in $\widehat{\mathbf{r}}_{\tau}$. The symmetry index is given by:

End For

$$
\mathrm{IND}_{\text {sym }}^{(\tau)}=\frac{1}{l} \sum_{i=1}^{l} \widehat{\mathbf{r}}_{\tau_{i}}
$$

Equivalent symmetry check:

$$
\mathrm{IND}_{\text {sym }}^{(\text {equ })}=\frac{1}{M} \sum_{i=1}^{M}\left|\operatorname{IND}_{\text {sym }}^{\left(\tau_{i}\right)}\right|<0 .
$$

Algorithm 2: Steps of the CAF symmetry-based detector.

The algorithm, illustrated in Figure 12, starts by splitting the band of the received signal in order to differentiate between the strong PU and other frequency components (SUs + distortions). The band splitting is accompanied by a coarse energy detector used to locate the strong interferer. Then a parallel block of reference nonlinearities is used to extract potential distortion products from the strong interferer. An adaptive filter, the least mean square (LMS), is used to adjust digitally created distortions levels. The adaptive filter utilizes the distorted signal resulted from the band splitter as an input parameter and minimizes the common error signal $e(t)$. The adjusted nonlinearities are finally subtracted from the received signal to cleanse the band from nonlinearity distortions. It is shown in [26] that the application of the AIC algorithm before the detector increases the detection reliability in CR devices.

3.3. Wideband Spectrum Sensing. Several emerging wireless applications and regulation encourage cognitive receivers to scan a wideband spectrum to find potential spectrum holes. In contrast to the narrowband techniques mentioned above, wideband spectrum sensing methods aim to sense a frequency bandwidth exceeding the coherence bandwidth of the channel. A frequent example deals with the design of an algorithm capable of sensing the whole ultra-high-frequency (UHF) TV band (between $300 \mathrm{MHz}$ and $3 \mathrm{GHz}$ ). Practically, wideband scanning could be performed via the following two different methods.

(1) By using a filter bank formed by preset multiple narrowband pass filters BPFs [105]. This hardware-based solution requires more hardware components, thus increasing the cost and the RF impairments harmful

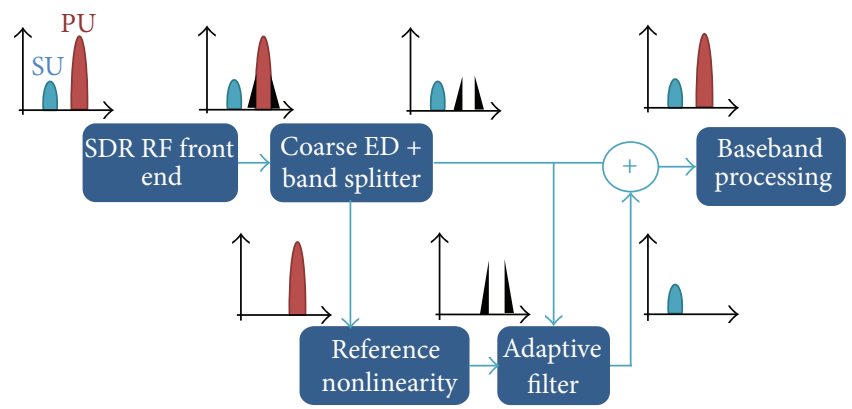

FIgURE 12: The AIC algorithm.

effect, and limiting the flexibility of the radio by fixing the number of filters. After each filter, a narrowband state-of-the-art technique is implemented.

(2) By using sophisticated signal processing techniques. In fact, narrowband sensing techniques cannot be directly applied to scan a wideband since they are based on single binary decision for the whole spectrum. Thus, they cannot simultaneously identify vacant channels that lie within the wideband spectrum. Recently proposed wideband spectrum sensing can be broadly categorized into two types:

(i) Nyquist wideband sensing processes digital signals taken at or above the Nyquist rate, for example, the wavelet transform-based technique;

(ii) sub-Nyquist wideband sensing acquires signals using a sampling rate lower than the Nyquist rate, for example, the compressive sensing technique. 


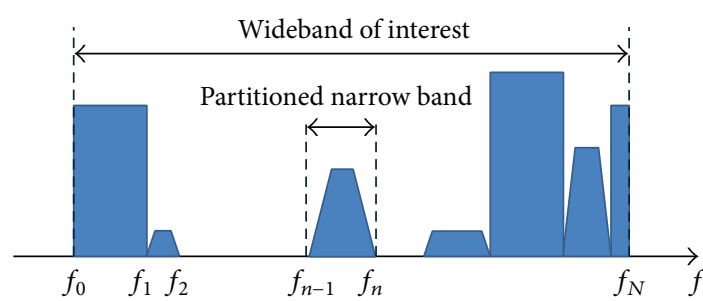

FIgURE 13: A wideband spectrum seen as a train of narrowband signals and presenting frequency irregularities.

In the following sections, two approaches to perform wideband spectrum sensing are discussed.

3.3.1. Wavelet Transform-Based Technique. In this method, the SU transceiver scans a wideband without using a bank of narrow BPFs. Alternatively, a wideband receiver will be based on high-speed digital signal processing to search over multiple frequency bands in an adaptive manner. The obtained digital signal will be modeled as a train of consecutive narrow frequency bands as illustrated in Figure 13. To identify these bands and search for potential spectrum holes, the wavelet transform will be used to locate edges between different narrow subbands [106]. The corresponding block diagram is depicted in Figure 14. Wavelet transform is used in mathematics to locate irregularities [95]. Consequently, it will be a good candidate to differentiate between the narrow subbands of wideband signal $[97,107]$. A wavelet edge detector is able to identify the average power level within each identified subband which will lead to the localization of the spectrum holes.

The analysis using wavelet transform is based on a function known as the principal wavelet $\psi$ which has a finite energy. Wavelets are used to transform a given signal into another representation that models the information related to the signal in a more utile way. Wavelets could be manipulated in two different ways: moved along the frequency axis or stretched with a variable energy. A Wavelet transform, obtained by summing the product of the signal multiplied by the wavelet, is calculated at different spots of the signal and for different combinations of the wavelet. This calculation could be monitored to detect the irregularities of the signal by observing the different values of the wavelet transform.

3.3.2. Compressive Sensing Technique. A major implementation challenge lies in the very high sampling rates required by conventional spectral estimation methods which have to operate at or above the Nyquist rate. However, to solve this issue, the compressive sampling CS technique is used for the acquisition of sparse signals at rates significantly lower than the Nyquist rate. Signal reconstruction is no more based on old reconstruction techniques but will be a solution to an optimization problem. Several schemes were suggested in the literature for the reconstruction of the signal, by using wavelet transforms [97], the autocorrelation of the signal [108], or advanced algorithms for sparse approximation methods [104, 109]. It is shown that such methods could preserve the adaptive response of the algorithm by offering a relatively small processing time.

Sparse approximation consists of finding a signal or a vector with sparseness property; that is, it has a small number of nonzero elements that satisfies (approximately) a system of equations. For example, consider a linear system of equations $y=\mathbf{A} x$, where $\mathbf{A}$ is an $n-$ by $-M$ matrix with $n<M$. Since A is overcomplete $(n<M)$, this problem does not have a unique solution. Among all the possible solutions, if the true one is known a priori to be sparse then it happens that the sparsest, that is, the solution $x$, containing as many as possible zero components and satisfying $y \cong A x$, is close to the true solution. Reducing the problem complexity to $n$ instead of $M$ increases the adaptation time of the reconstruction algorithm and thus provides better processing time.

\section{Machine Learning in Cognitive Radios}

Cognitive radios (CRs) are considered as intelligent radio devices that use the methodology of understanding-bybuilding to learn and adapt to their radio frequency (RF) environment [1]. Several CR architectures have been proposed over the past years in order to achieve dynamic spectrum access (DSA) [1-3]. However, as initially proposed by [4], the concept of CRs goes beyond DSA applications and aims to improve the quality of information (QoI) of wireless users [5]. This functionality requires an intelligent radio that is aware of its RF environment and is able to autonomously adapt to the variations in the wireless medium [9].

Cognitive radios are assumed to use spectrum sensing techniques to identify the RF activities in their surrounding environment [6]. Based on their observations, CRs apply their reasoning abilities to modify their behavior and adapt to particular situations. This is achieved through a reasoning engine which executes actions based on certain rules and strategies [27]. Similar reasoning engines could be identified in conventional radios that behave according to a set of hard-coded rules [27]. For example, according to the IEEE 802.11 specifications, such hard-coded rules determine the switching of a radio device among different modulation schemes depending on the signal-to-noise ratio (SNR) [27]. Hard-coded policies are completely specified by the system designer and may result in the desired performance as long as the operating conditions do not deviate from the original assumed model. However, in situations where the $\mathrm{RF}$ environment changes due to unexpected agents or factors (e.g., jammers, interferers, extreme fading conditions, etc.), the hard-coded rules may not lead to optimal performance, making them inefficient in this case. Cognitive radios, however, can overcome this limitation by updating their own sets of policies and rules based on past experience [27]. For example, if a $\mathrm{CR}$ is subject to jamming or significant interference on a certain channel, it could come up with new actions to switch to a new frequency band, instead of simply modifying its modulation scheme, in contrast with the IEEE 802.11 case. Hence, based on its learning ability, a CR can update or augment its set of rules and policies based on its own experience, which may lead to a more reliable 


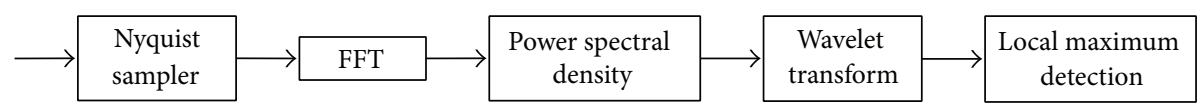

FiguRE 14: Block diagram of Wavelet transform-based technique.

communication performance $[9,27]$. This makes the learning ability a fundamental building block in any CR to achieve autonomous intelligent behavior [9, 27-29].

Machine learning techniques are gaining more importance in the current and future wireless networks due to the increase in system complexity and the heterogeneous nature of the wireless medium [29, 110-117]. In particular, significant research efforts are being focused on developing link aggregation techniques using multiple radio access networks (RANs) [111]. For example, the authors in [111] have demonstrated a CR system that is able to access a heterogeneous RAN aggregation system including HSDPA, Wi-Max, and WCDMA systems. In order to ensure efficient system operation, the system parameters need to be optimized to maximize the overall performance. However, due to the dynamic nature of the wireless network and the large number of system parameters, the operating parameters cannot be optimized manually, but require intelligent algorithms that are able to autonomously adjust the system parameters, leading to optimal performance. In [111], the support vector regressor (SVR)-based learning algorithm has been proposed for parameter optimization. This algorithm requires a set of training data to estimate the system model. Other learning approaches may be considered in the future to optimize various system parameters under different operating environments [111]. In this paper, however, we focus on unsupervised learning methods to ensure autonomous CR operation, as we will discuss next.

\section{The Cognitive Engine}

Cognitive radios extend software-defined radios (SDRs) by adding a cognitive engine (CE) to the radio platform [27]. According to [27], a CE can be composed of three main components: (1) a knowledge base, (2) a reasoning engine, and (3) a learning engine, as illustrated in Figure 15 [27]. The reasoning engine executes the actions and policies that are stored in the knowledge base, while the learning engine updates these policies based on past experience $[9,27]$. By applying learning algorithms, the learning engine can transform the observed data into knowledge, thus allowing the CR to be aware of certain characteristics of its environment $[4,29]$.

The machine learning literature is rich with learning algorithms that can be used in various contexts [29, 110-117]. These learning algorithms can be categorized under either supervised or unsupervised methods. In supervised learning, a set of labeled training data is available for the learning agent to specify whether a certain action is correct or wrong $[29,118]$. In unsupervised learning, however, the learning agent is supposed to identify the correct and wrong actions based on its own experience and interactions with the environment [118]. This makes unsupervised learning algorithms

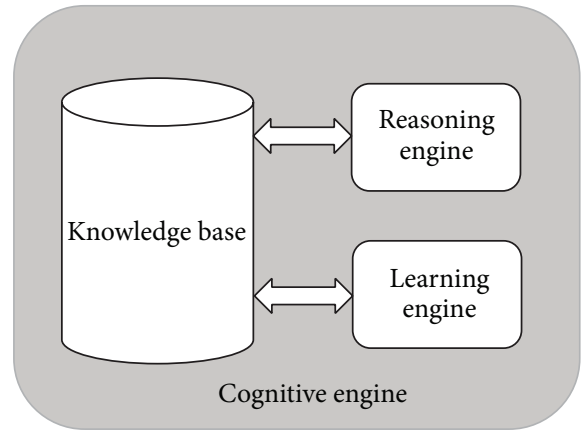

FIgURE 15: The cognitive engine (CE).

more appealing for $\mathrm{CR}$ applications, compared to supervised learning, since they lead to autonomous cognitive behavior in the absence of instructors [9]. Hence, unsupervised learning has been the focus of recent autonomous CRs formulations $[7,9,119-123]$.

Several unsupervised learning algorithms have been proposed for CRs to perform either feature classification or decision-making [29]. Classification algorithms can be used to infer hidden information about a set of noisy data. They allow, for example, inferring both number and types of wireless systems that are active in a certain environment [120, 124]. In addition, classification algorithms were also proposed for modulation classification based on Bayesian networks, as in [125]. On the other hand, decision-making algorithms can be used to update or modify the policies and rules that are stored in the knowledge base of a CR. Thus, the learning process may result in a new set of actions, allowing the CR to adapt to completely new RF environments [27].

In the followings, we present several unsupervised classification algorithms that have been proposed for autonomous signal classification in CRs. We also present a reinforcement learning (RL) algorithm that has been proposed to perform decision-making in CR networks.

5.1. Unsupervised Classification Algorithms. Classification algorithms have been proposed for CRs to extract knowledge from noisy data $[120,121,124]$. As we have mentioned above, the classification algorithms based on machine learning techniques can be divided into two main categories: upervised and unsupervised classifiers [29, 120]. Supervised classifiers require an "instructor" that specifies whether a certain classification decision is "correct" or not. These supervised classifiers require a set of labeled training data (or expertannotated data [120]) that specify the correct classes (or clusters). Supervised classifiers can be applied in certain conditions when prior knowledge (e.g., labeled data) is available to the learning agent. However, in situations where no such 
prior information is available, unsupervised classifiers can be used instead. Unsupervised classifiers do not require labeled data and process the detected data to determine the class or cluster of each data element. Hence, unsupervised classifiers can be considered more suitable for autonomous CRs when operating in unknown RF environments $[9,124]$.

In the followings, we present three examples of unsupervised classifiers that have been proposed for CRs: (1) the $K$-means, (2) $X$-means, and (3) Dirichlet process mixture model (DPMM) classifiers. We give a brief description of each algorithm and show their different applications in CRs.

5.1.1. The K-Means Classifier. The $K$-means algorithm has been proposed for robust signal classification in CRs [120]. It is considered as an unsupervised classifier since it does not require labeled training data $[29,120]$. However, the $K$ means algorithm requires prior knowledge about the number of clusters $K$, making it a unsupervised parametric classifier.

Given a set of $N$ feature points $\left\{\mathbf{y}_{i}\right\}_{i=1}^{N}$, the $K$-means algorithm classifies these data points into $K$ clusters, where $K$ is determined a priori. The algorithm starts with a set of $K$ arbitrary centroids $\left\{\mathbf{c}_{1}, \ldots, \mathbf{c}_{K}\right\}$, defining the centers of $K$ initial clusters. Each feature point $\mathbf{y}_{i}$ is then selected sequentially and assigned to the closest centroid (in terms of Euclidean distance). Once a feature point is assigned to a particular cluster, the corresponding centroid is updated and computed as the mean of the feature points belonging to that cluster. Eventually, the means converge to the clusters centers [120].

In $\mathrm{CR}$ applications, the $K$-means algorithm was applied to classify different types of RF signals based on their spectral or modulation characteristics. For example, in [120], the $K$-means were allowed to classify primary and secondary signals transmitting within the TV band. The authors in [120] demonstrated the robustness of this classifier against fluctuations in the signal parameters, such as the signalto-noise ratio (SNR). In general, such fluctuations lead to larger variance in the extracted features, which may cause poor classification performance. Nevertheless, the $K$-means algorithm was shown to be robust against such variations and achieved good performance in classifying both 8-level vestigial sideband (8VSB) modulated signals (primary TV transmission) and BPSK modulated signals (cognitive secondary transmission) [120].

The $K$-means algorithm is characterized by its low complexity and fast convergence. However, it requires accurate knowledge about the number of signal classes, which may not be practical in many cases $[9,120,124]$. For example, in many CR applications, it may be required to classify signals belonging to an unknown number of systems, which requires nonparametric approaches, as we will describe next $[9,119$, 120].

5.1.2. The $X$-Means Classifier. The $X$-means algorithm has been proposed as an extension of the $K$-means algorithm, allowing the classifier to estimate the number of clusters from the data itself [126]. The $X$-means algorithm is formulated as an iterative $K$-means algorithm which computes the optimal number of clusters that maximizes either the Bayesian information criterion (BIC) or Akaike information criterion (AIC) [126]. In contrast with the $K$-means, the $X$-means algorithm assumes an unknown number of clusters, making it suitable for nonparametric classification.

This algorithmic approach was successful in detecting primary user emulation (PUE) attacks in CR applications, as discussed in [120]. In this case, the signal detector can first estimate the number of clusters $X$ and then obtain the different classification regions for each cluster, similar to the $K$-means algorithm. Both $K$-means and $X$-means algorithms can be implemented at low complexity. However, they are suitable only for spherical Gaussian mixture models [124]. In CRs, however, feature vectors can be extracted from complex observation models which are not necessarily Gaussian [124]. This situation can thus be addressed using the DPMM classifier which assumes an arbitrary distribution of the observation model but requires higher computational complexity, compared to both $X$-means and $K$-means algorithms, as we will discuss next [124]:

$$
\theta_{i} \mid\left\{\theta_{j}\right\}_{j \neq i}, \mathbf{y}_{1}, \ldots, \mathbf{y}_{N}\left\{\begin{array}{l}
=\theta_{j} \quad \text { with prob. } \quad q_{j}=\frac{f_{\theta_{j}}\left(\mathbf{y}_{i}\right)}{\alpha_{0} f\left(\mathbf{y}_{i}\right)+\sum_{j=1, j \neq i}^{N} f_{\theta_{j}}\left(\mathbf{y}_{i}\right)} \\
\sim f\left(\theta_{i} \mid \mathbf{y}_{i}\right) \quad \text { with prob. } \quad q_{0}=\frac{\alpha_{0} f\left(\mathbf{y}_{i}\right)}{\alpha_{0} f\left(\mathbf{y}_{i}\right)+\sum_{j=1, j \neq i}^{N} f_{\theta_{j}}\left(\mathbf{y}_{i}\right)}
\end{array}\right.
$$

5.1.3. The DPMM Classifier. The DPMM classifier has been proposed for unsupervised signal classification in CRs [29, $121,124]$. It is considered a Bayesian nonparametric unsupervised classifier in the sense of allowing the number of clusters (or classes) to increase with the data size $[29,121,124]$. This model allows classifying a set of feature vectors $\left\{\mathbf{y}_{i}\right\}_{i=1}^{N}$ into $K$ clusters, where $K$ is to be estimated from the data (in contrast with the $K$-means which require prior knowledge about $K$ ).
The DPMM is based on the Chinese restaurant process (CRP) which models the feature vectors as customers joining specific tables [127]. The CRP has been previously proposed for both feature classification and decision-making [124, 128]. In particular, [128] proposed a strategic game model based on the CRP, which is referred to as the Chinese restaurant game. This framework has been applied for channel access in CR networks [128]. In this paper, on the other hand, 
we will present the CRP as an underlying framework for nonparametric signal classification in CRs, as in [124]. This model is formulated based on the DPMM, as discussed next.

The DPMM assumes that the feature vectors are drawn from a mixture model such that $[29,124,127]$

$$
\begin{gathered}
G \sim D P\left(\alpha_{0}, G_{0}\right) \\
\theta_{i} \mid G \sim G \\
\mathbf{y}_{i} \mid \theta_{i} \sim f_{\theta_{i}}\left(\mathbf{y}_{i}\right),
\end{gathered}
$$

where $G$ is a realization of the Dirichlet process $D P\left(\alpha_{0}, G_{0}\right)$ with parameters $\alpha_{0}>0$ and a prior distribution $G_{0}[29,124$, 127]. The nonparametric nature of the DPMM stems from the support distribution $G$ which is drawn from a nonparametric set of distributions according to the Dirichlet process. The realization $G \sim D P\left(\alpha_{0}, G_{0}\right)$ is discrete and defined over an infinite set, thus allowing for infinitely many clusters. Based on this model, a feature vector $\mathbf{y}_{i}$ is assumed to be drawn from a distribution $f_{\theta_{i}}\left(\mathbf{y}_{i}\right)$, where $\theta_{i}$ is drawn from $G[29,124]$. According to this formulation, a cluster is defined as a set of feature vectors $\mathbf{y}_{i}$ 's having identical parameters $\theta_{i}$ 's. Thus, we define the clusters parameters $\phi_{k}$ 's to denote the unique values of $\theta_{i}$ 's $[29,124,127]$.

By assuming the above DPMM framework, the problem of feature classification can be formulated following a Bayesian approach which estimates the parameters $\theta_{i}$ 's for a set of feature vectors $\left\{\mathbf{y}_{i}\right\}_{i=1}^{N}$, assuming a nonparametric prior $G \sim D P\left(\alpha_{0}, G_{0}\right)$ for $\theta_{i}$ 's $[129,130]$. The optimal parameters $\theta_{i}$ 's can thus be estimated based on the maximum a posteriori probability (MAP) criterion, which finds the parameters $\theta_{i}$ 's maximizing the posterior distribution of $f\left(\theta_{1}, \ldots, \theta_{N}\right.$ । $\left.\mathbf{y}_{1}, \ldots, \mathbf{y}_{N}\right)[124,129]$. However, this posterior distribution cannot be obtained in closed form under the above DPMM construction. Thus, stochastic simulation approaches have been proposed to estimate $\theta_{i}$ 's by using the Gibbs sampling method $[124,129]$. By following the Gibbs sampling approach, the DPMM-based classifier can be obtained by sampling $\theta_{i}$ 's from the posterior distribution $\theta_{i} \mid\left\{\theta_{j}\right\}_{j \neq i}, \mathbf{y}_{1}, \ldots, \mathbf{y}_{N}$ in (2) $[29,124,127,129,130]$.

This classification algorithm has been used for signal classification in CRs to determine the number of wireless systems in a certain RF environment $[121,124]$. It was shown to accurately estimate the number of existing wireless systems without any prior information about the environment. However, this algorithm requires extensive computational efforts since it relies on an iterative Gibbs sampling process.

5.2. Reinforcement Learning Algorithms. In addition to its ability of classifying wireless signals, a CR is assumed to use machine learning techniques for decision-making $[4,9$, 27, 29, 131-133]. This includes the ability to develop and adapt new strategies allowing us to maximize certain performance measures. In particular, the RL algorithms have been proposed to achieve such unsupervised decision-making in CRs [7, 29, 122, 123, 133-135]. The concept of RL is based on learning from experience by trial and error $[29,118]$. After executing a certain action, the learning agent receives a certain reward showing how good it is to take a particular action in a certain environment state [29, 118, 122]. As a result, the learning agent (the $\mathrm{CR}$, in this case) will select certain actions that lead to the highest rewards in a particular state. The corresponding action selection method is based on an exploration-exploitation strategy that selects the highest reward action with a higher probability, compared to the other available actions [118]. This can be usually implemented using the $\epsilon$-greedy approach which selects a greedy action with a probability $1-\epsilon$ and a random action with small probability $\epsilon$, thus allowing us to avoid local optima $[7,118]$.

The Q-learning algorithm is one of the $\mathrm{RL}$ algorithms that has been proposed for CR applications [7, 118, 122]. Under a Markov decision process (MDP) framework, the Q-learning can lead to optimal policy, yet without knowledge of the state transition probabilities $[136,137]$. An MDP is characterized by the following elements [7, 29, 122, 133, 137]:

(i) a finite set $\mathcal{S}$ of states for the agent (i.e., secondary user);

(ii) a finite set $\mathscr{A}$ of actions that are available to the agent;

(iii) a nonnegative function $p_{t}\left(s^{\prime} \mid s, a\right)$ denoting the probability that the system is in state $s^{\prime}$ at time epoch $t+1$, when the decision-maker chooses action $a \in \mathscr{A}$ in state $s \in \mathcal{S}$ at time $t$;

(iv) a real-valued function $r_{t}^{\mathrm{MDP}}(s, a)$ defined for state $s \in$ $\mathcal{S}$ and action $a \in \mathscr{A}$ to denote the value at time $t$ of the reward received in period $t$ [137].

At each time epoch $t$, the agent observes the current state $s$ and chooses an action $a$. The objective is to find the optimal policy $\pi$ that maximizes the expected discounted return [118]:

$$
R(t)=\sum_{k=0}^{\infty} \gamma^{k} r_{t+k+1}\left(s_{t+k}, a_{t+k}\right),
$$

where $s_{t}$ and $a_{t}$ are, respectively, the state and action at time $t \in Z$.

The optimal policy of the MDP can be based on the Qfunction (or action-value function) which determines how good it is to take a particular action $a$ in a given state $s$. Formally, the Q-function is defined as the value of taking action $a$ in state $s$ under a policy $\pi$ [118]:

$$
Q^{\pi}(s, a)=\mathbb{E}_{\pi}\left\{R(t) \mid s_{t}=s, a_{t}=a\right\} .
$$

This function can be computed using an iterative procedure as follows $[7,29,122,133,136]$ :

$$
\begin{aligned}
Q\left(s_{t}, a_{t}\right) \longleftarrow & (1-\alpha) Q\left(s_{t}, a_{t}\right) \\
& +\alpha\left[r_{t+1}\left(s_{t}, a_{t}\right)+\gamma \max _{a} Q\left(s_{t+1}, a\right)\right] .
\end{aligned}
$$

The RL algorithm can be represented in the block diagram of Figure 16 in which the learning agent receives the state observation $o_{t}$ and the reward function $r_{t}$ at each instant $t[29,118]$. It then updates its $Q$-function at the learning stage and selects an appropriate action $a_{t}$. Under the MDP assumption, the $Q$-learning can guarantee convergence of the Q-function to its optimal value [136]. Thus, the optimal policy 


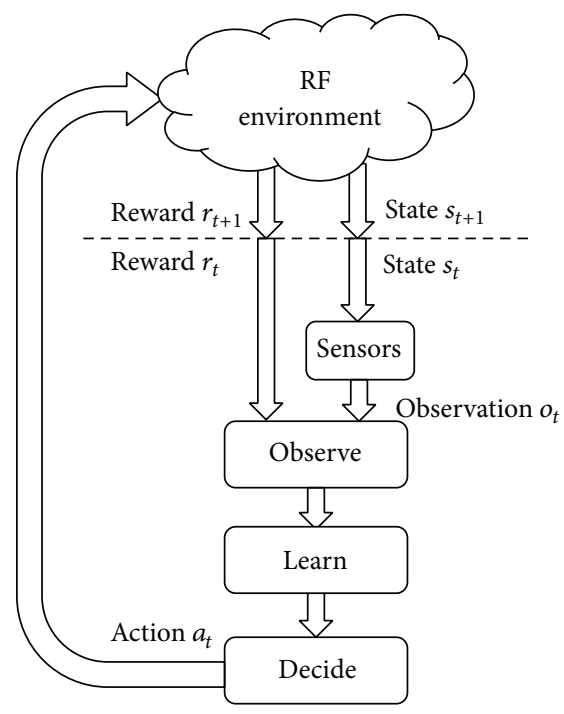

Figure 16: The RL cycle.

$\pi$ can be obtained in function of the $Q$-function such that $[29,118]$

$$
a^{*}(s)= \begin{cases}\arg \max _{a \in \mathscr{A}} Q(s, a), & \text { with } \operatorname{Pr}=1-\varepsilon \\ \sim U(\mathscr{A}), & \text { with } \operatorname{Pr}=\varepsilon,\end{cases}
$$

where $U(\mathscr{A})$ is the discrete uniform probability distribution over the set of actions $\mathscr{A}$ and $a^{*}(s)$ is the optimal action selected in state $s$ and corresponding to the optimal policy $\pi$.

The Q-learning algorithm has been proposed for two main CR applications:

(1) aggregate interference control $[8,122]$;

(2) spectrum sensing policy [7, 123].

In aggregate interference control, the Q-learning was proposed to optimize the power transmission of secondary CRs in a WRAN IEEE 802.22 CR network (CRN) [122]. The objective is to maintain the aggregated interference caused by the secondary networks to the DTV network below a certain threshold. In this scenario, the CRs constitute a distributed network and each radio tries to determine how much power it can transmit so that the aggregated interference on the primary receivers does not exceed a certain threshold level [122]. Simulation results have shown that the Q-learning algorithm can successfully control the aggregate interference in a WRAN scenario [122].

On the other hand, the Q-learning algorithm has been proposed for opportunistic spectrum access (OSA) applications to coordinate the actions of CRs in a distributed CRN [7]. Given a set of CRs, the Q-learning can determine the channel that should be sensed by each CR at each time instant in order to maximize the average utilization of idle primary channels while limiting collision among secondary cognitive users [7]. The Q-learning algorithm was shown to achieve near-optimal performance in such OSA applications [7]. However, it cannot guarantee optimal policy for the decentralized partially observable decision-making problem, which is considered one of the most challenging problems for MDPs, in general $[137,138]$. However, given the limited amount of information, the Q-learning algorithm can be considered as one of the most effective low-complexity approaches for distributed partially observable decisionmaking scenarios $[7,122,133]$.

\section{Conclusion}

This paper presented a review of three major front-end CR elements: the RF part, spectrum sensing, and machine learning. For the RF part, three types of antennas were presented: UWB antennas, used for spectrum sensing, frequencyreconfigurable/tunable antennas for communicating over white spaces (also for sequential channel sensing), and UWB antennas with reconfigurable band notches for overlay UWB $\mathrm{CR}$. Also for the RF part, it was shown that the main design challenges are those pertaining to the ADCs/DACs, dynamic range, LNAs, filters, mixers, and synthesizers. Sophisticated spectrum sensing algorithms that overcome the challenges resulting from the adaptive behavior of CR transceivers need to be developed to relax RF designs and provide accurate decisions. A CE executes actions based on certain rules and policies that are learnt from past experience. With the growing complexity of the current wireless networks, more sophisticated learning algorithms should be developed, taking into account the heterogeneous structure of existing and future communication networks.

\section{Conflict of Interests}

The authors declare that there is no conflict of interests regarding the publication of this paper.

\section{References}

[1] S. Haykin, "Cognitive radio: brain-empowered wireless communications," IEEE Journal on Selected Areas in Communications, vol. 23, no. 2, pp. 201-220, 2005.

[2] A. Goldsmith, S. A. Jafar, I. Maric, and S. Srinivasa, "Breaking spectrum gridlock with cognitive radios: an information theoretic perspective," Proceedings of the IEEE, vol. 97, no. 5, pp. 894-914, 2009.

[3] N. Devroye, M. Vu, and V. Tarokh, "Cognitive radio networks: highlights of information theoretic limits, models, and design," IEEE Signal Processing Magazine, vol. 25, no. 6, pp. 12-23, 2008.

[4] J. Mitola III and G. Q. Maguire Jr., "Cognitive radio: making software radios more personal," IEEE Personal Communications, vol. 6, no. 4, pp. 13-18, 1999.

[5] J. Mitola III, "Cognitive radio architecture evolution," Proceedings of the IEEE, vol. 97, no. 4, pp. 626-641, 2009.

[6] T. Yücek and H. Arslan, "A survey of spectrum sensing algorithms for cognitive radio applications," IEEE Communications Surveys and Tutorials, vol. 11, no. 1, pp. 116-130, 2009.

[7] M. Bkassiny, S. K. Jayaweera, and K. A. Avery, "Distributed Reinforcement Learning based MAC protocols for autonomous cognitive secondary users," in Proceedings of the 20th Annual Wireless and Optical Communications Conference (WOCC '11), pp. 1-6, Newark, NJ, USA, April 2011. 
[8] L. Giupponi, A. Galindo-Serrano, P. Blasco, and M. Dohler, "Docitive networks: an emerging paradigm for dynamic spectrum management," IEEE Wireless Communications, vol. 17, no. 4, pp. 47-54, 2010.

[9] S. K. Jayaweera and C. G. Christodoulou, "Radiobots: architecture, algorithms and realtime reconfigurable antenna designs for autonomous, self-learning future cognitive radios," Tech. Rep. EECE-TR-11-0001, University of New Mexico, 2011, http://repository.unm.edu/handle/1928/12306.

[10] A. Sonnenschein and P. M. Fishman, "Radiometric detection of spread-spectrum signals in noise of uncertain power," IEEE Transactions on Aerospace and Electronic Systems, vol. 28, no. 3, pp. 654-660, 1992.

[11] S. M. Kay, Fundamentals of Statistical Signal Processing: Detection Theory, Prentice-Hall, Upper Saddle River, NJ, USA, 1998.

[12] Z. Ye, G. Memik, and J. Grosspietsch, "Energy detection using estimated noise variance for spectrum sensing in cognitive radio networks," in Proceedings of the IEEE Wireless Communications and Networking Conference (WCNC '08), pp. 711-716, Las Vegas, Nev, USA, March-April 2008.

[13] H. S. Chen, W. Gao, and D. G. Daut, "Signature based spectrum sensing algorithms for IEEE 802.22 WRAN," in Proceedings of the IEEE International Conference on Communications (ICC '07), pp. 6487-6492, Glasgow, UK, June 2007.

[14] S. Xu, Z. Zhao, and J. Shang, "Spectrum sensing based on cyclostationarity," in Proceedings of the IEEE Workshop on Power Electronics and Intelligent Transportation System (PEITS '08), pp. 171-174, Guangzhou, China, August 2008.

[15] W. A. Gardner, "Exploitation of spectral redundancy in cyclostationary signals," IEEE Signal Processing Magazine, vol. 8, no. 2, pp. 14-36, 1991.

[16] W. A. Gardner, A. Napolitano, and L. Paura, "Cyclostationarity: half a century of research," Signal Processing, vol. 86, no. 4, pp. 639-697, 2006.

[17] W. A. Gardner, "Spectral correlation of modulated signals-part I: analog modulation," IEEE Transactions on Communications, vol. 35, no. 6, pp. 584-595, 1987.

[18] W. A. Gardner, W. A. Brown III, and C. Chen, "Spectral correlation of modulated signals-part II: digital modulation," IEEE Transactions on Communications, vol. 35, no. 6, pp. 595601, 1987.

[19] Y. H. Zeng and Y. C. Liang, "Covariance based signal detections for cognitive radio," in Proceedings of the 2nd IEEE International Symposium on New Frontiers in Dynamic Spectrum Access Networks (DySPAN '07), pp. 202-207, Dublin, Ireland, April 2007.

[20] Y. H. Zeng and Y. C. Liang, "Spectrum-sensing algorithms for cognitive radio based on statistical covariances," IEEE Transactions on Vehicular Technology, vol. 58, no. 4, pp. 1804-1815, 2009.

[21] Y. H. Zeng and Y. C. Liang, "Eigenvalue based sensing algorithms," IEEE 802. 22-06/0118r0, 2006.

[22] Y. H. Zeng and Y. C. Liang, "Maximum-minimum eigenvalue detection for cognitive radio," in Proceedings of the 18th Annual IEEE International Symposium on Personal, Indoor and Mobile Radio Communications (PIMRC '07), Athens, Greece, September 2007.

[23] Y. H. Zeng and Y. C. Liang, "Eigenvalue-based spectrum sensing algorithms for cognitive radio," IEEE Transactions on Communications, vol. 57, no. 6, pp. 1784-1793, 2009.

[24] G. Fettweis, M. Lohning, D. Petrovic, M. Windisch, P. Zillmann, and W. Rave, "Dirty RF: a new paradigm," in Proceedings of the IEEE 16th International Symposium on Personal, Indoor and Mobile Radio Communications (PIMRC '05), vol. 4, pp. 23472355, Berlin, Germany, September 2005.

[25] M. Valkama, A. S. H. Ghadam, L. Anttila, and M. Renfors, "Advanced digital signal processing techniques for compensation of nonlinear distortion in wideband multicarrier radio receivers," IEEE Transactions on Microwave Theory and Techniques, vol. 54, no. 6, pp. 2356-2366, 2006.

[26] M. Grimm, R. K. Sharma, M. A. Hein, and R. S. Thomä, “DSPbased mitigation of RF front-end non-linearity in cognitive wideband receivers," Frequenz, vol. 6, no. 9-10, pp. 303-310, 2012.

[27] C. Clancy, J. Hecker, E. Stuntebeck, and T. O’Shea, "Applications of machine learning to cognitive radio networks," IEEE Wireless Communications, vol. 14, no. 4, pp. 47-52, 2007.

[28] S. K. Jayaweera, Y. Li, M. Bkassiny, C. Christodoulou, and K. A. Avery, "Radiobots: the autonomous, self-learning future cognitive radios," in Proceedings of the International Symposium on Intelligent Signal Processing and Communication Systems (ISPACS '11), pp. 1-5, Chiang Mai, Thailand, December 2011.

[29] M. Bkassiny, Y. Li, and S. K. Jayaweera, "A survey on machinelearning techniques in cognitive radios," IEEE Communications Surveys Tutorials, vol. 15, no. 3, pp. 1136-1159, 2013.

[30] M. Al-Husseini, K. Y. Kabalan, A. El-Hajj, and C. G. Christodoulou, "Reconfigurable microstrip antennas for cognitive radio," in Advancement in Microstrip Antennas with Recent Applications, A. Kishk, Ed., chapter 14, pp. 337-362, InTech, Rijeka, Croatia, 2013.

[31] P. Samal, P. Soh, and G. Vandenbosch, "A systematic design procedure for microstrip-based unidirectional UWB antennas," Progress In Electromagnetics Research, vol. 143, pp. 105-130, 2013.

[32] B. Gong, J. Li, Q. R. Zheng, Y. Z. Yin, and X. S. Ren, "A compact inductively loaded monopole antenna for future UWB applications," Progress In Electromagnetics Research, vol. 139, pp. 265-275, 2013.

[33] W. Liu, Y. Yin, W. Xu, and S. Zuo, "Compact open-slot antenna with bandwidth enhancement," IEEE Antennas and Wireless Propagation Letters, vol. 10, pp. 850-853, 2011.

[34] M.-R. Ghaderi and F. Mohajeri, "A compact hexagonal wide-slot antenna with microstrip-fed monopole for UWB application," IEEE Antennas and Wireless Propagation Letters, vol. 10, pp. 682-685, 2011.

[35] H. Oraizi and S. Hedayati, "Miniaturized UWB monopole microstrip antenna design by the combination of Giusepe Peano and Sierpinski carpet fractals," IEEE Antennas and Wireless Propagation Letters, vol. 10, pp. 67-70, 2011.

[36] P. C. Ooi and K. T. Selvan, "The effect of ground plane on the performance of a square loop CPW-fed printed antenna," Progress In Electromagnetics Research Letters, vol. 19, pp. 103-111, 2010.

[37] M. Al-Husseini, A. Ramadan, Y. Tawk, A. El-Hajj, and K. Y. Kabalan, "Design and ground plane consideration of a CPWFed UWB antenna," in Proceedings of the International Conference on Electrical and Electronics Engineering (ELECO '09), pp. II-151-II-153, Bursa, Turkey, November 2009.

[38] M. Al-Husseini, A. Ramadan, A. El-Hajj, and K. Kabalan, "Design of a compact and low-cost fractal-based UWB PCB Antenna," in Proceedings of the National Radio Science Conference (NRSC '09), pp. 1-8, Cairo, Egypt, March 2009.

[39] A. Mehdipour, K. Mohammadpour-Aghdam, R. Faraji-Dana, and M.-R. Kashani-Khatib, "A novel coplanar waveguide-fed 
slot antenna for ultrawideband applications," IEEE Transactions on Antennas and Propagation, vol. 56, no. 12, pp. 3857-3862, 2008.

[40] M. Al-Husseini, A. El-Hajj, and K. Y. Kabalan, "A 1.9-13.5 GHz low-cost microstrip antenna," in Proceedings of the International Wireless Communications and Mobile Computing Conference (IWCMC'08), pp. 1023-1025, Crete Island, Greece, August 2008.

[41] Z. N. Low, J. H. Cheong, and C. L. Law, "Low-cost PCB antenna for UWB applications," IEEE Antennas and Wireless Propagation Letters, vol. 4, no. 1, pp. 237-239, 2005.

[42] M. Al-Husseini, Y. Tawk, A. El-Hajj, and K. Y. Kabalan, "A low-cost microstrip antenna for 3G/WLAN/WiMAX and UWB applications," in Proceedings of the International Conference on Advances in Computational Tools for Engineering Applications (ACTEA '09), pp. 68-70, Zouk Mosbeh, Lebanon, July 2009.

[43] M. Al-Husseini, A. Ramadan, Y. Tawk, A. El-Hajj, and K. Y. Kabalan, "Design and ground plane optimization of a CPW-fed ultra-wideband antenna," Turkish Journal of Electrical Engineering and Computer Sciences, vol. 19, no. 2, pp. 243-250, 2011.

[44] H. Zhang, X. Zhou, and T. Chen, "Ultra-wideband cognitive radio for dynamic spectrum accessing networks," in Cognitive Radio Networks, Y. Xiao and F. Hu, Eds., pp. 353-382, CRC Press, Boca Raton, Fla, USA, 2009.

[45] L. Safatly, M. Al-Husseini, A. El-Hajj, and K. Kabalan, "Advanced techniques and antenna design for pulse shaping in UWB cognitive radio," International Journal of Antennas and Propagation, vol. 2012, Article ID 390280, 8 pages, 2012.

[46] J. B. Pendry, A. J. Holden, D. J. Robbins, and W. J. Stewart, "Magnetism from conductors and enhanced nonlinear phenomena," IEEE Transactions on Microwave Theory and Techniques, vol. 47, no. 11, pp. 2075-2084, 1999.

[47] F. Falcone, T. Lopetegi, M. A. G. Laso et al., "Babinet principle applied to the design of metasurfaces and metamaterials," Physical Review Letters, vol. 93, no. 19, Article ID 197401, 4 pages, 2004.

[48] P. Moeikham, C. Mahatthanajatuphat, and P. Akkaraekthalin, "A compact uwb antenna with a quarter-wavelength strip in a rectangular slot for $5.5 \mathrm{GHz}$ band notch," International Journal of Antennas and Propagation, vol. 2013, Article ID 574128, 9 pages, 2013.

[49] B. Yan, D. Jiang, R. M. Xu, and Y. H. Xu, "A UWB band-pass antenna with triple-notched band using common direction rectangular complementary split-ring resonators," International Journal of Antennas and Propagation, vol. 2013, Article ID 934802, 6 pages, 2013.

[50] X. Liu, Y.-Z. Yin, P. Liu, J. Wang, and B. Xu, "A CPW-fed dual bandnotched UWB antenna with a pair of bended dual-L-shape parasitic branches," Progress In Electromagnetics Research, vol. 136, pp. 623-634, 2013.

[51] R. Azim and M. Islam, "Compact planar UWB antenna with band notch characteristics for WLAN and DSRC," Progress In Electromagnetics Research, vol. 133, pp. 391-406, 2013.

[52] J. R. Kelly, P. S. Hall, and P. Gardner, "Band-notched UWB antenna incorporating a microstrip open-loop resonator," IEEE Transactions on Antennas and Propagation, vol. 59, no. 8, pp. 3045-3048, 2011.

[53] T.-D. Nguyen, D.-H. Lee, and H.-C. Park, "Design and analysis of compact printed triple band-notched UWB antenna," IEEE Antennas and Wireless Propagation Letters, vol. 10, pp. 403-406, 2011.

[54] M. Almalkawi and V. Devabhaktuni, "Ultrawideband antenna with triple band-notched characteristics using closed-loop ring resonators," IEEE Antennas and Wireless Propagation Letters, vol. 10, pp. 959-962, 2011.

[55] D.-O. Kim, N.-I. Jo, H.-A. Jang, and C.-Y. Kim, "Design of the ultrawideband antenna with a quadruple-band rejection characteristics using a combination of the complementary split ring resonators," Progress In Electromagnetics Research, vol. 112, pp. 93-107, 2011.

[56] Y. Li, W. Li, and Q. Ye, "A reconfigurable triple-notch-band antenna integrated with defected microstrip structure band-stop filter for ultrawideband cognitive radio applications," International Journal of Antennas and Propagation, vol. 2013, Article ID 472645, 13 pages, 2013.

[57] T. Aboufoul, A. Alomainy, and C. Parini, "Reconfigured and notched tapered slot UWB antenna for cognitive radio applications," International Journal of Antennas and Propagation, vol. 2012, Article ID 160219, 8 pages, 2012.

[58] J. Perruisseau-Carrier, P. Pardo-Carrera, and P. Miskovsky, "Modeling, design and characterization of a very wideband slot antenna with reconfigurable band rejection," IEEE Transactions on Antennas and Propagation, vol. 58, no. 7, pp. 2218-2226, 2010.

[59] C.-Y. Sim, W.-T. Chung, and C.-H. Lee, "Planar uwb antenna with $5 \mathrm{ghz}$ band rejec-tion switching function at ground plane," Progress In Electromagnetics Research, vol. 106, pp. 321-333, 2010.

[60] M. Al-Husseini, J. Costantine, C. G. Christodoulou, S. E. Barbin, A. El-Hajj, and K. Y. Kabalan, "A reconfigurable frequencynotched UWB antenna with split-ring resonators," in Proceedings of the Asia-Pacific Microwave Conference (APMC '10), pp. 618-621, Yokohama, Japan, December 2010.

[61] M. Al-Husseini, A. Ramadan, A. El-Hajj, K. Y. Kabalan, Y. Tawk, and C. G. Christodoulou, "Design based on complementary split-ring resonators of an antenna with controllable band notches for UWB cognitive radio applications," in Proceedings of the IEEE International Symposium on Antennas and Propagation (APSURSI), pp. 1120-1122, Spokane, Wash, USA, July 2011.

[62] M. Al-Husseini, L. Safatly, A. Ramadan, A. El-Hajj, K. Y. Kabalan, and C. G. Christodoulou, "Reconfigurable filter antennas for pulse adaptation in UWB cognitive radio systems," Progress In Electromagnetics Research B, no. 37, pp. 327-342, 2011.

[63] E. Ebrahimi, J. R. Kelly, and P. S. Hall, "Integrated wide-narrowband antenna for multi-standard radio," IEEE Transactions on Antennas and Propagation, vol. 59, no. 7, pp. 2628-2635, 2011.

[64] G. Augustin and A. Denidni, "An integrated ultra wideband/ narrow band antenna in uniplanar configuration for cognitive radio systems," IEEE Transactions on Antennas and Propagation, vol. 60, no. 11, pp. 5479-5484, 2012.

[65] M. Al-Husseini, Y. Tawk, C. G. Christodoulou, A. El Hajj, and K. Y. Kabalan, "A reconfigurable cognitive radio antenna design," in Proceedings of the IEEE Antennas and Propagation Society International Symposium (APSURSI), pp. 1-4, Toronto, Canada, July 2010.

[66] M. R. Hamid, P. Gardner, P. S. Hall, and F. Ghanem, "Switchedband Vivaldi antenna," IEEE Transactions on Antennas and Propagation, vol. 59, no. 5, pp. 1472-1480, 2011.

[67] M. R. Hamid, P. Gardner, P. S. Hall, and F. Ghanem, "Vivaldi antenna with integrated switchable band pass resonator," IEEE Transactions on Antennas and Propagation, vol. 59, no. 11, pp. 4008-4015, 2011.

[68] T. Aboufoul, A. Alomainy, and C. Parini, "Reconfiguring UWB monopole antenna for cognitive radio applications using GaAs FET switches," IEEE Antennas and Wireless Propagation Letters, vol. 11, pp. 392-394, 2012. 
[69] M. Al-Husseini, A. Ramadan, M. E. Zamudio, C. G. Christodoulou, A. El-Hajj, and K. Y. Kabalan, "A UWB antenna combined with a reconfigurable bandpass filter for cognitive radio applications," in Proceedings of the IEEE-APS Topical Conference on Antennas and Propagation in Wireless Communications (APWC '11), pp. 902-904, Torino, Italy, September 2011.

[70] Y. Cai, Y. Guo, and T. Bird, "A frequency reconfigurable printed yagiuda dipole antenna for cognitive radio applications," IEEE Transactions on Antennas and Propagation, vol. 60, no. 6, pp. 2905-2912, 2012.

[71] M. Y. A. Shahine, M. Al-Husseini, Y. Nasser, K. Y. Kabalan, and A. El-Hajj, "A reconfigurable miniaturized spiral monopole antenna for TV white spaces," in Proceedings of the 34th Progress in Electromagnetics Research Symposium (PIERS '13), Stockholm, Sweden, August 2013.

[72] G. Mansour, P. Hall, P. Gardner, and M.-K. A. Rahim, “Tunable slotloaded patch antenna for cognitive radio," in Proceedings of the Loughborough Antennas and Propagation Conference (LAPC '12), Loughborough, UK, November 2012.

[73] Y. Tawk, M. E. Zamudio, J. Costantine, and C. Christodoulou, "A cognitive radio reconfigurable 'filtenna," in Proceedings of the 6th European Conference on Antennas and Propagation (EUCAP '12), pp. 3565-3568, Prague, Czech Republic, March 2012.

[74] V. Nguyen, F. Villain, and Y. Le Guillou, "Cognitive radio RF: overview and challenges," VLSI Design, 2012.

[75] M. Kitsunezuka, K. Kunihiro, and M. Fukaishi, "Efficient use of the spectrum," IEEE Microwave Magazine, vol. 13, no. 1, pp. 5563, 2012.

[76] B. Razavi, "Cognitive radio design challenges and techniques," IEEE Journal of Solid-State Circuits, vol. 45, no. 8, pp. 1542-1553, 2010.

[77] J. Arias, L. Quintanilla, J. Segundo, L. Enríquez, J. Vicente, and J. M. Hernández-Mangas, "Parallel continuous-time $\Delta \Sigma$ ADC for OFDM UWB receivers," IEEE Transactions on Circuits and Systems I, vol. 56, no. 7, pp. 1478-1487, 2009.

[78] C. Lelandais-Perrault, T. Petrescu, D. Poulton, P. Duhamel, and J. Oksman, "Wideband, bandpass, and versatile hybrid filter bank A/D conversion for software radio," IEEE Transactions on Circuits and Systems I, vol. 56, no. 8, pp. 1772-1782, 2009.

[79] C.-C. Huang, C.-Y. Wang, and J.-T. Wu, "A CMOS 6-Bit 16-GS/s time-interleaved ADC using digital background calibration techniques," IEEE Journal of Solid-State Circuits, vol. 46, no. 4, pp. 848-858, 2011.

[80] A. Gruget, M. Roger, V. T. Nguyen, C. Lelandais-Perrault, P. Bénabès, and P. Loumeau, "Wide-band multipath A to D converter for cognitive radio applications," in Proceedings of the IEEE International Microwave Workshop Series on RF Front-ends for Software Defined and Cognitive Radio Solutions (IMWS '10), pp. 73-76, Aveiro, Portugal, February 2010.

[81] A. Gruget, M. Roger, V. T. Nguyen, C. Lelandais-Perrault, P. Bénabès, and P. Loumeau, "Optimization of bandpass charge sampling filters in hybrid filter banks converters for cognitive radio applications," in Proceedings of the 20th European Conference on Circuit Theory and Design (ECCTD '11), pp. 785-788, Linköping, Sweden, August 2011.

[82] Z. Boos, A. Menkhoff, F. Kuttner et al., "A fully digital multimode polar transmitter employing $17 \mathrm{~b}$ RF DAC in $3 \mathrm{G}$ mode," in Proceedings of the IEEE International Solid-State Circuits Conference (ISSCC '11), pp. 376-377, San Francisco, Calif, USA, February 2011.

[83] A. Pozsgay, T. Zounes, R. Hossain, M. Boulemnakher, V. Knopik, and S. Grange, "A fully digital $65 \mathrm{~nm}$ CMOS transmitter for the 2.4-to-2.7GHz WiFi/WiMAX bands using $5.4 \mathrm{GHz} \delta \sigma \mathrm{RF}$ DACs," in Proceedings of the IEEE International Solid State Circuits Conference (ISSCC '08), pp. 355-619, San Francisco, Calif, USA, February 2008.

[84] J. Borremans, G. Mandal, V. Giannini et al., "A $40 \mathrm{~nm}$ CMOS $0.4-6 \mathrm{GHz}$ receiver resilient to out-of-band blockers," IEEE Journal of Solid-State Circuits, vol. 46, no. 7, pp. 1659-1671, 2011.

[85] M. C. M. Soer, E. A. M. Klumperink, Z. Ru, F. E. van Vliet, and B. Nauta, "A 0.2-to-2.0 GHz $65 \mathrm{~nm}$ CMOS receiver without LNA achieving $>11 \mathrm{dBm}$ IIP3 and $<6.5 \mathrm{~dB}$ NF", in Proceedings of the IEEE International Solid-State Circuits Conference (ISSCC '09), pp. 222-223, San Francisco, Calif, USA, February 2009.

[86] C. Andrews and A. C. Molnar, "A passive mixer-first receiver with digitally controlled and widely tunable RF interface," IEEE Journal of Solid-State Circuits, vol. 45, no. 12, pp. 2696-2708, 2010.

[87] A. Mirzaie, A. Yazdi, Z. Zhou, E. Chang, P. Suri, and H. Darabi, "A $65 \mathrm{~nm}$ CMOS quad-band SAW-less receiver for GSM/ GPRS/EDGE," in Proceedings of the Symposium on VLSI Circuits (VLSIC '10), pp. 179-180, Honolulu, Hawaii, USA, June 2010.

[88] A. Ghaffari, E. A. M. Klumperink, M. C. M. Soer, and B. Nauta, "Tunable high-q N-Path Band-Pass filters: modeling and verification," IEEE Journal of Solid-State Circuits, vol. 46, no. 5, pp. 998-1010, 2011.

[89] S. C. Blaakmeer, E. A. M. Klumperink, B. Nauta, and D. M. W. Leenaerts, "An inductorless wideband balun-LNA in $65 \mathrm{~nm}$ CMOS with balanced output," in Proceedings of the 33rd European Solid-State Circuits Conference (ESSCIRC '07), pp. 364367, Munich, Germany, September 2007.

[90] Z. Ru, N. A. Moseley, E. A. M. Klumperink, and B. Nauta, “Digitally enhanced software-defined radio receiver robust to outof-band interference," IEEE Journal of Solid-State Circuits, vol. 44, no. 12, pp. 3359-3375, 2009.

[91] A. A. Rafi, A. Piovaccari, P. Vancorenland, and T. Tuttle, "A harmonic rejection mixer robust to RF device mismatches," in Proceedings of the IEEE International Solid-State Circuits Conference (ISSCC '11), pp. 66-68, San Francisco, Calif, USA, February 2011.

[92] J. Borremans, K. Vengattaramane, V. Giannini, B. Debaillie, W. van Thillo, and J. Craninckx, "A $86 \mathrm{MHz}-12 \mathrm{GHz}$ digital-intensive PLL for software-defined radios, using a $6 \mathrm{fJ} / \mathrm{step}$ TDC in $40 \mathrm{~nm}$ digital CMOS," IEEE Journal of Solid-State Circuits, vol. 45, no. 10, pp. 2116-2129, 2010.

[93] J. Mitola, Cognitive radio: an integrated agent architecture for software defined radio [Ph.D. thesis], Royal Institute of Technology, KTH, 2000.

[94] Z. Quan, S. Cui, A. H. Sayed, and H. V. Poor, "Optimal multiband joint detection for spectrum sensing in cognitive radio networks," IEEE Transactions on Signal Processing, vol. 57, no. 3, pp. 1128-1140, 2009.

[95] Z. Tian and G. B. Giannakis, "A wavelet approach to wideband spectrum sensing for cognitive radios," in Proceedings of the 1st International Conference on Cognitive Radio Oriented Wireless Networks and Communications (CROWNCOM '06), pp. 1-5, Mykonos Island, Greece, June 2006.

[96] W. Liu and S. Ding, "Multiuser detections based on global optimality necessary conditions for binary quadratic programming," in Proceedings of the 2nd International Conference on Innovative Computing, Information and Control (ICICIC '07), Kumamoto, Japan, September 2007.

[97] Z. Tian and G. B. Giannakis, "Compressed sensing for wideband cognitive radios," in Proceedings of the IEEE International 
Conference on Acoustics, Speech and Signal Processing (ICASSP '07), vol. 4, pp. IV-1357-IV-1360, Honolulu, Hawaii, USA, April 2007.

[98] E. G. Larsson and M. Skoglund, "Cognitive radio in a frequency-planned environment: some basic limits," IEEE Transactions on Wireless Communications, vol. 7, no. 12, pp. 4800-4806, 2008.

[99] A. Sahai, N. Hoven, and R. Tandra, "Some fundamental limits on cognitive radio," in Proceedings of the 42nd Annual Allerton Conference on Communication, Control, and Computing, pp. 1662-1671, Monticello, Ill, USA, October 2004.

[100] R. Tandra and A. Sahai, "SNR walls for signal detection," IEEE Journal on Selected Topics in Signal Processing, vol. 2, no. 1, pp. $4-17,2008$.

[101] E. G. Larsson and G. Regnoli, "Primary system detection for cognitive radio: does small-scale fading help?" IEEE Communications Letters, vol. 11, no. 10, pp. 799-801, 2007.

[102] I. M. Johnstone, "On the distribution of the largest eigenvalue in principal components analysis," Annals of Statistics, vol. 29, no. 2, pp. 295-327, 2001.

[103] Z. Khalaf, A. Nafkha, and J. Palicot, "Blind spectrum detector for cognitive radio using compressed sensing and symmetry property of the second order cyclic autocorrelation," in Proceedings of the 7th International ICST Conference on Cognitive Radio Oriented Wireless Networks and Communications (CROWNCOM '12), pp. 291-296, Stockholm, Sweden, June 2012.

[104] G. Davis, S. Mallat, and M. Avellaneda, "Adaptive greedy approximations," Constructive Approximation, vol. 13, no. 1, pp. 5798, 1997.

[105] A. Sahai and D. Cabric, "Spectrum sensing: fundamental limits and practical challenges," in Proceedings of the IEEE International Symposium on New Frontiers in Dynamic Spectrum Access Networks (DySPAN '05), 2005.

[106] H. Sun, A. Nallanathan, C. X. Wang, and Y. Chen, "Wideband spectrum sensing for cognitive radio networks: a survey," IEEE Wireless Communications, vol. 20, no. 2, pp. 74-81, 2013.

[107] S. Chantaraskul and K. Moessner, "Implementation of wavelet analysis for spectrum opportunity detection," in Proceedings of the IEEE 20th Personal, Indoor and Mobile Radio Communications Symposium (PIMRC '09), pp. 2310-2314, Tokyo, Japan, September 2009.

[108] Y. L. Polo, Y. Wang, A. Pandharipande, and G. Leus, "Compressive wide-band spectrum sensing," in Proceedings of the IEEE International Conference on Acoustics, Speech, and Signal Processing (ICASSP '09), pp. 2337-2340, Taipei City, Taiwan, April 2009.

[109] S. G. Mallat and Z. Zhang, "Matching pursuits with time-frequency dictionaries," IEEE Transactions on Signal Processing, vol. 41, no. 12, pp. 3397-3415, 1993.

[110] Y. Zhao and L. Morales-Tirado, "Cognitive radio technology: principles and practice," in Proceedings of the International Conference on Computing, Networking and Communications (ICNC '12), pp. 650-654, Maui, Hawaii, USA, February 2012.

[111] Y. Kon, M. Ito, N. Hassel, M. Hasegawa, K. Ishizu, and H. Harada, "Autonomous parameter optimization of a heterogeneous wireless network aggregation system using machine learning algorithms," in Proceedings of the IEEE Consumer Communications and Networking Conference (CCNC '12), pp. 894-898, Las Vegas, Nev, USA, January 2012.

[112] P. Venkatraman, B. Hamdaoui, and M. Guizani, "Opportunistic bandwidth sharing through reinforcement learning," IEEE
Transactions on Vehicular Technology, vol. 59, no. 6, pp. 31483153, 2010.

[113] J. Oksanen, J. Lundén, and V. Koivunen, "Reinforcement learning method for energy efficient cooperative multiband spectrum sensing," in Proceedings of the IEEE International Workshop on Machine Learning for Signal Processing (MLSP '10), pp. 59-64, Kittila, Finland, September 2010.

[114] Z. Chen and R. C. Qiu, "Q-learning based bidding algorithm for spectrum auction in cognitive radio," in Proceedings of the IEEE SoutheastCon 2011, pp. 409-412, Nashville, Tenn, USA, March 2011.

[115] K.-L.A. Yau, P. Komisarczuk, and P. D. Teal, "Performance analysis of reinforcement learning for achieving context awareness and intelligence in mobile cognitive radio networks," in Proceedings of the IEEE International Conference on Advanced Information Networking and Applications (AINA '11), pp. 1-8, Singapore, March 2011.

[116] P. Venkatraman and B. Hamdaoui, "Cooperative Q-learning for multiple secondary users in dynamic spectrum access," in Proceedings of the 7th International Wireless Communications and Mobile Computing Conference (IWCMC '11), pp. 238-242, Istanbul, Turkey, July 2011.

[117] D. Kumar, N. Kanagaraj, and R. Srilakshmi, "Harmonized qlearning for radio resource management in lte based networks," in Proceedings of ITU Kaleidoscope: Building Sustainable Communities, 2013.

[118] R. S. Sutton and A. G. Barto, Reinforcement Learning: An Introduction, MIT Press, Cambridge, Mass, USA, 1998.

[119] M. Bkassiny, S. K. Jayaweera, Y. Li, and K. A. Avery, "Wideband spectrum sensing and non-parametric signal classification for autonomous self-learning cognitive radios," IEEE Transactions on Wireless Communications, vol. 11, no. 7, pp. 2596-2605, 2012.

[120] T. C. Clancy, A. Khawar, and T. R. Newman, "Robust signal classification using unsupervised learning," IEEE Transactions on Wireless Communications, vol. 10, no. 4, pp. 1289-1299, 2011.

[121] N. Shetty, S. Pollin, and P. Pawelczak, "Identifying spectrum usage by unknown systems using experiments in machine learning," in Proceedings of the IEEE Wireless Communications and Networking Conference (WCNC '09), pp. 1-6, Budapest, Hungary, April 2009.

[122] A. Galindo-Serrano and L. Giupponi, "Distributed Q-learning for aggregated interference control in cognitive radio networks," IEEE Transactions on Vehicular Technology, vol. 59, no. 4, pp. 1823-1834, 2010

[123] K.-L. A. Yau, P. Komisarczuk, and P. D. Teal, "Applications of reinforcement learning to cognitive radio networks," in Proceedings of the IEEE International Conference on Communications Workshops, ICC 2010, pp. 1-6, Capetown, South Africa, May 2010.

[124] M. Bkassiny, S. K. Jayaweera, and Y. Li, "Multidimensional Dirichlet process-based non-parametric signal classification for autonomous selflearning cognitive radios," IEEE Transactions on Wireless Communications, vol. 12, no. 11, pp. 5413-5423, 2013.

[125] C. Phelps and R. Buehrer, "Signal classification by probabilistic reasoning," in Proceedings of the IEEE Radio and Wireless Symposium (RWS '13), pp. 154-156, Austin, Tex, USA, January 2013.

[126] D. Pelleg and A. Moore, "X-means: extending k-means with efficient estimation of the number of clusters," in Proceedings of the 7th International Conference on Machine Learning (ICML '00), Stanford, Calif, USA, June-July 2000. 
[127] Y. W. Teh, M. I. Jordan, M. J. Beal, and D. M. Blei, "Hierarchical Dirichlet processes," Journal of the American Statistical Association, vol. 101, no. 476, pp. 1566-1581, 2006.

[128] C.-Y. Wang, Y. Chen, and K. Liu, "Sequential Chinese restaurant game," IEEE Transactions on Signal Processing, vol. 61, no. 3, pp. 571-584, 2013.

[129] M. D. Escobar and M. West, "Bayesian density estimation and inference using mixtures," Journal of the American Statistical Association, vol. 90, no. 430, pp. 577-588, 1995.

[130] M. D. Escobar, "Estimating normal means with a dirichlet process prior," Journal of the American Statistical Association, vol. 89, no. 425, pp. 268-277, 1994.

[131] J. Mitola, Cognitive radio: an integrated agent architecture for software defined radio [Ph.D. thesis], KTH, 2000.

[132] V. Stavroulaki, A. Bantouna, Y. Kritikou et al., "Knowledge management toolbox: machine learning for cognitive radio networks," IEEE Vehicular Technology Magazine, vol. 7, no. 2, pp. 91-99, 2012.

[133] L. Gavrilovska, V. Atanasovski, I. Macaluso, and L. DaSilva, "Learning and reasoning in cognitive radio networks," IEEE Communications Surveys and Tutorials, vol. 15, no. 4, pp. 17611777, 2013.

[134] Y. Li, S. K. Jayaweera, M. Bkassiny, and C. Ghosh, "Learningaided sub-band selection algorithms for spectrum sensing in wide-band cognitive radios," IEEE Transactions on Wireless Communications. In press.

[135] Y. Li, S. K. Jayaweera, C. Ghosh, and M. Bkassiny, "Learningaided sensing scheduling for wide-band cognitive radios," in Proceedings of the IEEE 78th Vehicular Technology Conference (VTC Fall '13), pp. 1-5, Las Vegas, Nev, USA, September 2013.

[136] C. J. C. H. Watkins and P. Dayan, “Q-learning," Machine Learning, vol. 8, no. 3-4, pp. 279-292, 1992.

[137] M. L. Puterman, Markov Decision Processes: Discrete Stochastic Dynamic Programming, John Wiley \& Sons, New York, NY, USA, 1994.

[138] L. Buşoniu, R. Babuška, and B. de Schutter, "Multi-agent reinforcement learning: a survey," in Proceedings of the 9th International Conference on Control, Automation, Robotics and Vision (ICARCV '06), pp. 1-6, Singapore, December 2006. 

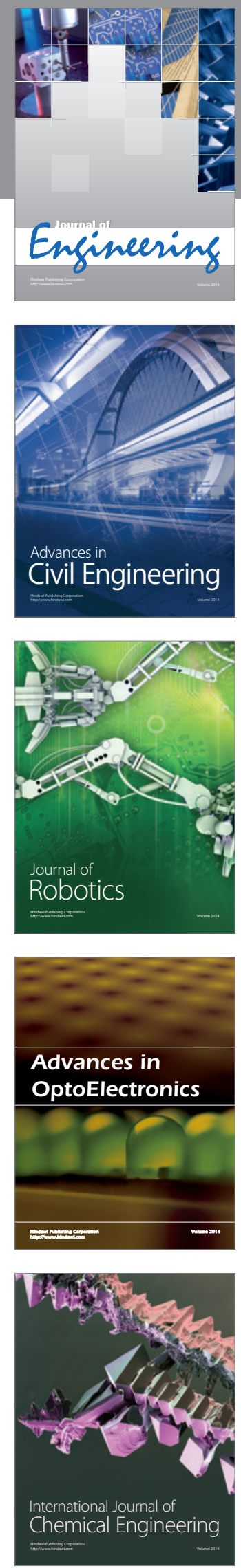

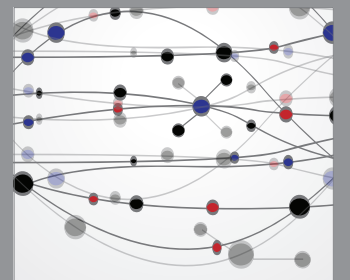

The Scientific World Journal
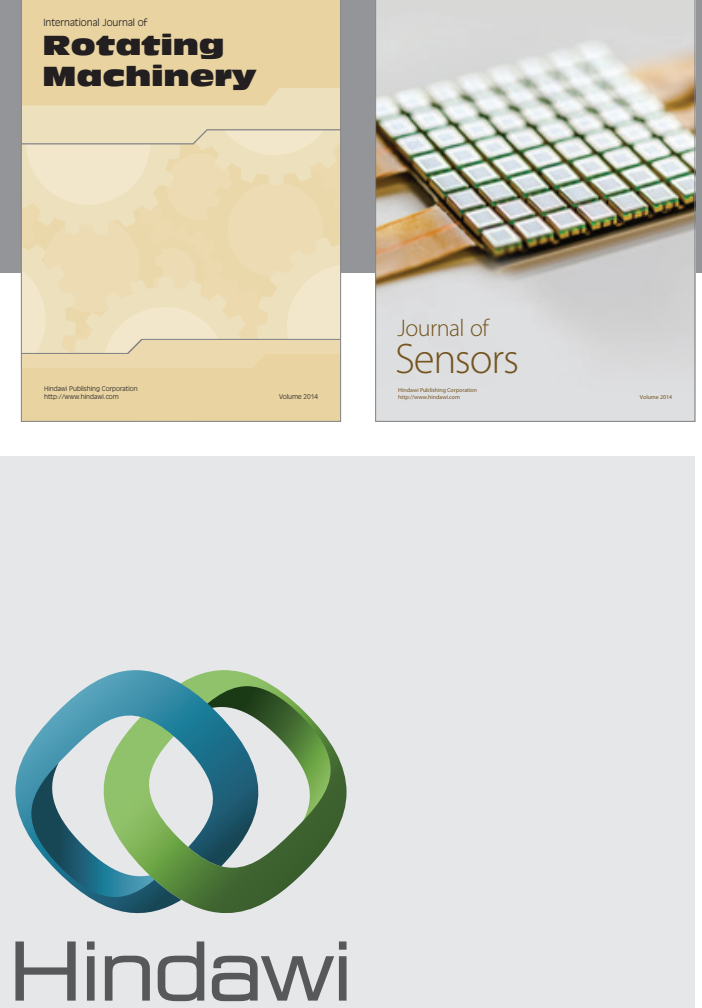

Submit your manuscripts at http://www.hindawi.com
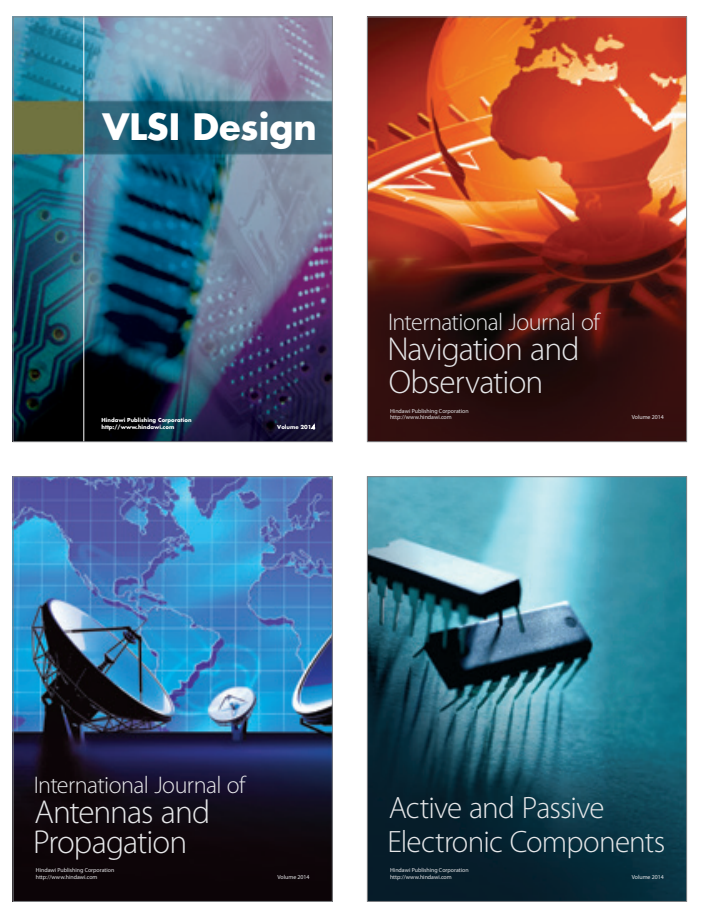
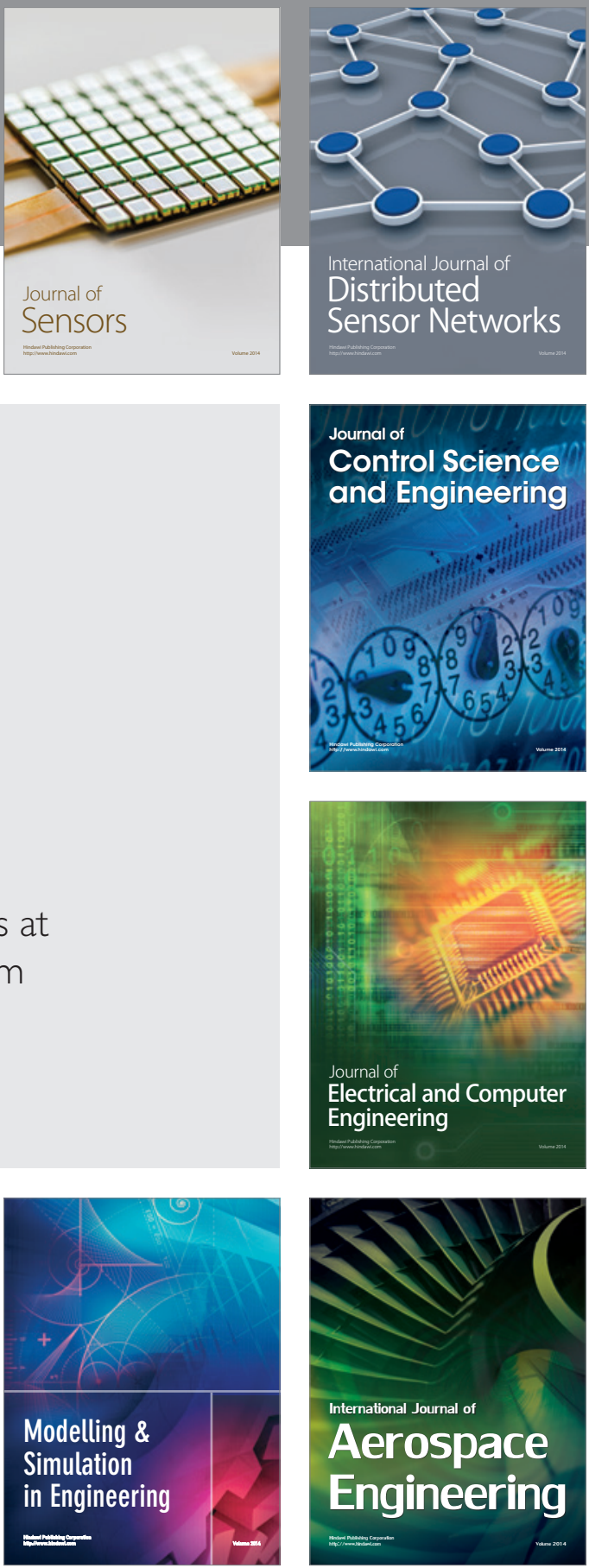

Journal of

Control Science

and Engineering
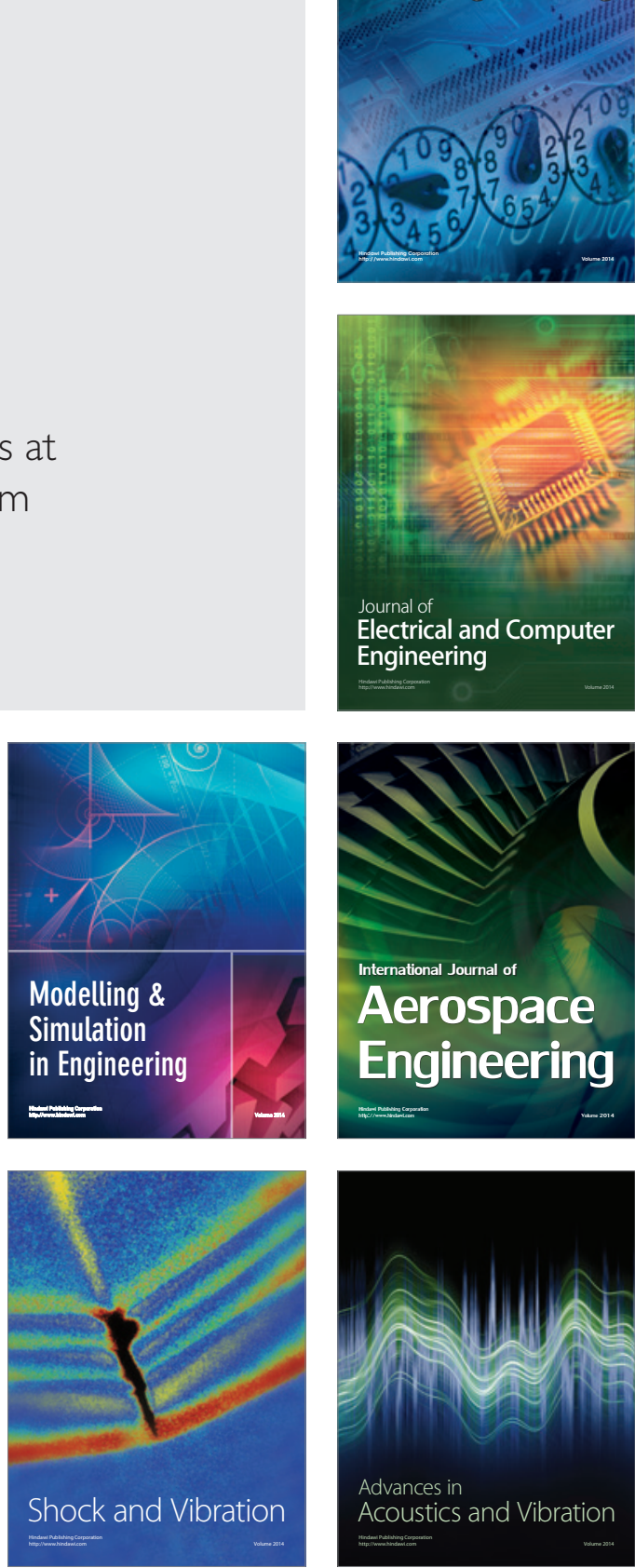